\title{
Magnetic structure and frequency scaling of limit-cycle oscillations close to L- to H-mode transitions
}

\author{
G. Birkenmeier, ${ }^{1,2}{ }^{*}$ M. Cavedon, ${ }^{1,2}$ G. D. Conway, ${ }^{2}$ P. Manz, ${ }^{1,2}$ U. Stroth, ${ }^{2}$ R. Fischer, ${ }^{2}$ G. Fuchert ${ }^{2}$ \\ T. Happel, ${ }^{2}$ F. M. Laggner, ${ }^{3}$ M. Maraschek, ${ }^{2}$ A. Medvedeva, ${ }^{1,2}$ V. Nikolaeva,,${ }^{1}$ D. Prisiazhniuk, ${ }^{1,2}$ T. Pütterich, ${ }^{2}$ \\ F. Ryter, ${ }^{2}$ L. M. Shao, ${ }^{2}$ M. Willensdorfer, ${ }^{2}$ E. Wolfrum,${ }^{2}$ H. Zohm,${ }^{2}$ and the ASDEX Upgrade Team \\ ${ }^{1}$ Physik-Department E28, Technische Universität München, 85748 Garching, Germany \\ ${ }^{2}$ Max Planck Institute for Plasma Physics,..Boltzmannstr. 2, 85748 Garching, Germany \\ ${ }^{3}$ Institute of Applied Physics, TU Wien, Fusion@ÖAW, Wiedner Hauptstr. 8-10, 1040 Vienna, Austria \\ ${ }^{4}$ Instituto de Plasmas e Fusão Nuclear, Instituto Superior Técnico, \\ Universidade Técnica de Lisboa, 1049-001 Lisboa, Portugal
}

\begin{abstract}
Limit-cycle oscillations (LCOs) close to the power threshold of L- to H-mode transitions are investigated in plasmas of ASDEX Upgrade. During this phase, referred to as I-phase, a strong magnetic activity in the poloidal magnetic field $\dot{B}_{\theta}$ with an up-down asymmetry is found. In some cases, the regular LCOs during I-phase transition smoothly into a phase with intermittent bursts which have similar properties to type-III edge localised modes (ELMs). Indications of precursors during the intermittent phase as well as in the regular LCO phase point to a common nature of the I-phase and type-III ELMs. The LCO frequency measured in a set of discharges with different plasma currents and magnetic fields scales as $f \sim\left(B_{t}^{1 / 2} I_{p}^{3 / 2}\right) /(n T)$.
\end{abstract}

PACS numbers: $52.25 . \mathrm{Xz}, 52.25 . \mathrm{Fi}, 52.35 . \mathrm{Ra}, 52.55 . \mathrm{Fa}$

\section{INTRODUCTION}

At the transition from low confinement (L-mode) to high confinement (H-mode) regimes in magnetically confined plasmas, regular pulsations in the low kilohertz range can occur at the edge of the plasma, for example visible in the edge $H_{\alpha}$-signal or divertor shunt currents [1]. Due to their non-sinusoidal shape and characteristic phase lags between two pulsing quantities, for example the density fluctuation level and poloidal flow velocity, these pulsations are referred to as limit-cycle oscillations (LCOs) since solutions of coupled differential equations as known from dynamical systems theory exhibit similar features.

The LCOs close to the transition from L- to H-mode (L-H transition) were investigated experimentally with various diagnostics, and depending on the interpretation of the investigating author, the LCO state was labelled differently. At ASDEX Upgrade (AUG) the LCO state was initially called 'dithering H-mode' and described as a modulation of electron temperature $T_{e}$ and density $n_{e}$, which could qualitatively be understood in terms of a model involving a bifurcation of the radial electric field inducing a bistable state toggling between the L- and $\mathrm{H}-$ mode [2]. Further studies on AUG with Doppler reflectometry [3] and a divertor X-point probe [4] were focusing on the role of flow-turbulence interactions inside and outside the last closed flux surface (LCFS) and used the term 'I-phase' in order to express the intermediate character of the LCO state sharing properties of both the L-mode and the H-mode. The intermediate character was also

\footnotetext{
*gregor.birkenmeier@ipp.mpg.de
}

documented in slow L- to H-transitions at DIII-D in the so called 'IM-mode' which is a very smooth transition from almost sinusoidal LCOs into a bursty phase identified as the type-III edge localised mode (ELM) phase $[5,6]$. Further reports on LCOs in EAST which evolve into typical type-III ELM phases with magnetic precursors can be found in references [7, 8]. Type-III ELMs are associated with a magnetic precursor activity, which is typically not detected during LCOs. This article addresses the question whether both pulsating phenomena originate from the same underlying physical mechanism. LCOs with a very specific magnetic structure and frequency scaling called 'M-mode' also have been found in JET discharges [9, 10], and many further studies at various types of tokamak configurations report on pulsations close to improved confinement regimes [11-13]. It is remarkable that the LCOs appear with similar properties even in non-axisymmetric configurations $[14,15]$.

While it is in general accepted that the final H-mode state is sustained by the neoclassical mean flow $[3,7,16$ 18 ] there is still some discussion whether the zonal flow-turbulence interaction dominates the LCO dynamics and therefore triggers the transition into improved confinement regimes. Several works report on increased Reynolds-stress work during the LCOs or at the transition to H-mode indicating that zonal flows are responsible for the turbulence suppression at the L-H transition [8, 19-23]. In addition, the ability of zonal flows to extract energy from turbulence, which effectively suppresses turbulence, is experimentally well documented in basic plasma experiments [24-26]. However, in some experiments at JFT-2M [12] and HL-2A [16] the Reynoldsstress drive was found to be too weak to explain the additional $E \times B$-flow which is necessary to trigger the L-H transition. Recent measurements in AUG plasmas sug- 

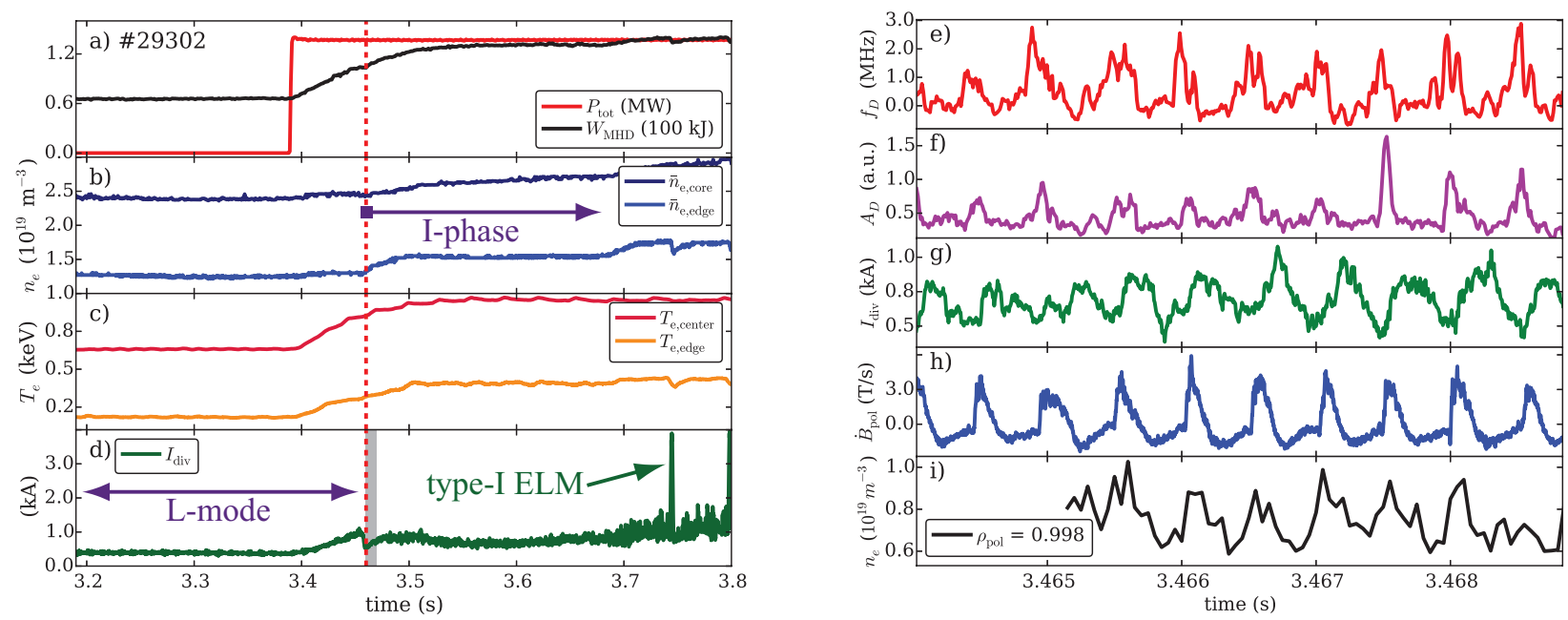

FIG. 1: Typical L-H transition at low density in AUG: After switching on the auxiliary heating $P_{\text {tot }}$, the plasma energy content $W_{\text {MHD }}(\mathrm{a})$ and the temperature (c) rise. The rise in line averaged density (b) starts with a delay of about 50 ms and coincides with a drop in the divertor shunt current (d) and the start of the LCOs (dashed line). The time traces (e)-(i) show a short segment of the LCOs and correspond to the shaded area in (d). The LCOs are visible in the Doppler shift $f_{D} \sim u_{\perp}$ (e) and the backscattered amplitude $A_{D} \sim \tilde{n}^{2}$ (f) of the Doppler reflectometry signal, as well as in the divertor current (g), magnetic probe signal below the divertor $(\mathrm{h})$, and the local density slightly inside the LCFS (i).

gest that the radial electric field $E_{r}$ agrees well with the electric field $E_{\mathrm{r}, \text { neo }}$ predicted by neoclassical theory at the transition from L-mode to I-phase (L-I transition) [27]. The agreement of $E_{r}$ with $E_{\mathrm{r}, \text { neo }}$ holds likewise throughout the evolution of the I-phase indicating a negligible contribution of zonal flows. This ambiguity of results from different experiments is unsatisfactory and partially caused by the lack of simultaneous measurements of neoclassical and turbulent contributions to the mean flow with sufficient temporal and spatial resolution.

The experimentally well documented LCOs can qualitatively be described with zero-dimensional models based on a zonal flow-turbulence interaction mediated by the Reynolds-stress resulting in predator-prey type of equations [28, 29]. This type of models has been extended by adding one or more 'predators' [30], it is formulated in a one-dimensional version to account for radial profile changes [31] and a recent version also includes electronion coupling in order to agree with the experimentally found minimum of the L- to $\mathrm{H}$-mode power threshold (L-H power threshold) [32]. This type of models based on zonal flow-turbulence interaction is, however, not the only set of equations with LCO solutions similar to experimental findings. LCO models derived from particle and heat transport equations $[2,33]$ or momentum transport equations [34] without involving zonal flows can likewise describe the experimental findings. In addition, models derived purely from magnetohydrodynamic (MHD) equations can produce the same LCOs dynamics [35], and several other models, mainly derived for the description of ELM cycles, have LCO solutions (for a review see e.g. reference [36]).
Due to their simplicity, the different types of LCO models contain heuristic terms or free parameters which allow in some cases to freely adjust the model solutions to the experimental situation. Therefore, a falsification of the models is difficult and it is not always obvious how to test the assumptions which enter into the derivation of the models.

Nevertheless, it is possible to compare the LCO frequency scaling as found in the experiments with the dependencies predicted by the models, and further measured characteristics can be used to identify the validity of model assumptions. In this paper, we report on the magnetic structure and the frequency scaling of LCOs based on measurements of magnetic pick up coils in a set of low power AUG plasmas at different magnetic fields and plasma currents. The results point to electromagnetic processes involved in the LCO dynamics and indicate a common physical mechanism of LCOs and type-III ELMs.

After a brief overview of the appearance of LCOs at the L-H transition (Section II) and at the back transition from H- to L-mode in AUG plasmas (Section III), we will discuss the spatial structure (Section IV) and precursor dynamics (Section V) of the LCOs as they appear in the magnetic signals. Finally, we will present the frequency dependence of LCOs in Section VI and discuss its implications for LCO models in Section VII. 


\section{LCOS AT THE L-H TRANSITION}

As already described in references [2, 3, 37], the I-phase at the forward transition from L- to H-mode appears in AUG close to the $\mathrm{L}-\mathrm{H}$ power threshold at line averaged densities below $5 \cdot 10^{19} \mathrm{~m}^{-3}$ and features frequencies from 0.5 to $5 \mathrm{kHz}$. Surprisingly, the I-phase appears in deuterium plasmas almost exclusively in favourable magnetic configurations (i.e. ion $\mathbf{B} \times \boldsymbol{\nabla} B$-drift towards the $\mathrm{X}$-point). The few examples of LCOs in unfavourable configuration are mainly observed at the back transition from $\mathrm{H}$ - to L-mode. The I-phase lasts longer for slow power ramps, but even in very fast power ramps at least a few LCO pulses can be identified. Hence, the LCOs (or at least some bursts) appear practically in every Lto $\mathrm{H}$-transition in favourable magnetic configuration. A typical L- to H-transition at low line averaged density $\bar{n}_{e} \approx 2.5 \cdot 10^{19} \mathrm{~m}^{-3}$, toroidal field $B_{t}=-2.5 \mathrm{~T}$, plasma current $I_{p}=0.6 \mathrm{MA}$, lower single null configuration, and low neutral beam heating power is shown in figure 1 .

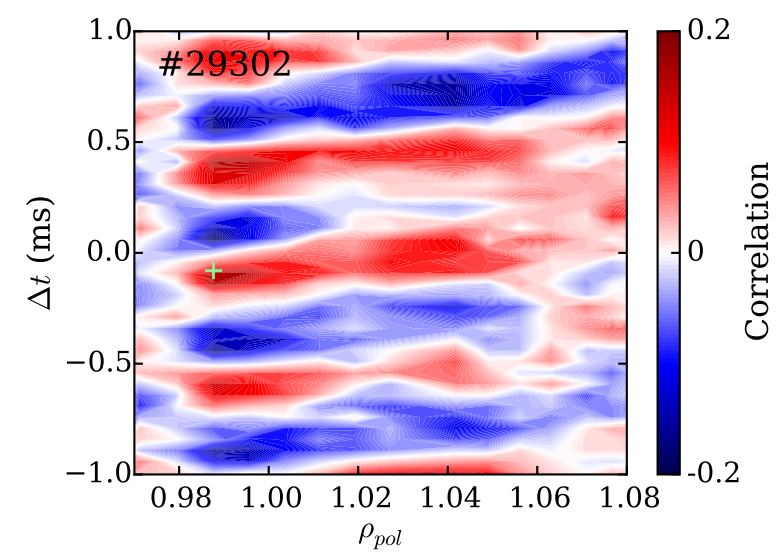

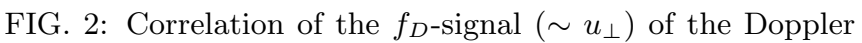
reflectometer with several radial channels of the Li-BES system $\left(\sim n_{e}\right)$ during the I-phase of discharge \#29302. The local maximum of the cross-correlation starts slightly inside the separatrix and propagates outward.

When the neutral beam heating is switched on, the plasma energy content $W_{\mathrm{MHD}}$ (determined from the equilibrium) and the temperature in the center $T_{e, c e n t e r}$ and at the edge $T_{\text {e,edge }}$ measured with the electron cyclotron emission (ECE) [38] system start immediately to rise (figure $1(\mathrm{c})$ ). The line averaged density $\bar{n}_{e}$ however stays initially at the same level and increases suddenly about 50 ms after switching on the heating power (figure 1(b)). At the same time when the density starts to rise, the divertor shunt current signal $I_{\text {div }}$ (figure $1(\mathrm{~d})$ ) drops significantly indicating reduced edge transport. This is the time point when the $2 \mathrm{kHz}$ modulation of the LCOs becomes clearly visible in many signals as shown in figure 1(e)-(i), although some weak oscillations can sometimes be observed before (the so-called small-amplitude LCOs as described in [7] but also reported in $[4,12,13])$. While the transition from L-mode to I-phase is fast (within some microseconds) and sharp, the 'transition' from I-phase to $\mathrm{H}$-mode is soft. In general, it is difficult to decide when the plasma entered the $\mathrm{H}$-mode state since the improved confinement is already achieved at the L-I transition as indicated by the rise in density and drop of the divertor current. A clear sign of a developed H-mode pedestal is the appearance of a type-I ELM as visible in the divertor current (figure $1(\mathrm{~d})$ ).

The LCOs after the drop of the divertor current exhibit the typical oscillation pattern visible in signals of many diagnostics. The V-band Doppler reflectometer system $[39,40]$ operated in X-mode, detects the LCOs in the Doppler shift $f_{D}$, which is proportional to the perpendicular flow velocity $u_{\perp}$ (figure $1(\mathrm{e})$ ), and in the modulated backscattered amplitude $A_{D}$, which is proportional to the power of the density fluctuation $\tilde{n}^{2}$ (figure $1(\mathrm{f})$ ) at the probing wavenumber $k_{\perp} \approx 9 \mathrm{~cm}^{-1}$. A microwave frequency of $68.5 \mathrm{GHz}$ was chosen corresponding to a radial position of $\rho_{\text {pol }} \approx 0.995$ in terms of the normalised poloidal flux coordinate $\rho_{\text {pol }}$, i.e. close to the $E_{r}$ minimum inside the LCFS. A clear indication of a geodesic acoustic mode (GAM) in frequency spectra as reported in reference [3] can not be found here possibly due to collisional damping at higher densities which are considered here. However, a few oscillations of the $f_{D}$ signal in accordance with the GAM frequency are typically observed during the I-phase bursts.

The LCO pulses are likewise visible in the divertor shunt current $I_{\text {div }}$ (figure $1(\mathrm{~g})$ ) and in a magnetic pick-up coil located below the inner divertor (coil no. 23 in figure $7\left(\right.$ b) ) measuring the poloidal field fluctuation $\dot{B}_{\theta}$ (figure $1(\mathrm{~h})$ ). The latter signal shows the LCOs most clearly and is in addition available in practically every plasma scenario. Therefore, we will make full use of it in the following. The density profiles measured by lithium beam emission spectroscopy (Li-BES) $[41,42]$ with a time resolution of $50 \mu$ s are likewise modulated in a radial region from $1 \mathrm{~cm}$ inside the LCFS to the far scrape-off layer. The associated modulation of the density gradient implicates a variation of the neoclassical radial electric field $E_{\mathrm{r}, \text { neo }}$ which in its simplest form is given by $\nabla p_{i} /\left(e n_{i}\right)$ with $\nabla p_{i}$ the main ion pressure gradient. In a detailed study [27] based on charge exchange recombination spectroscopy measurements, it was found that the measured $E_{\mathrm{r}}$ agrees very well with the neoclassical $E_{\mathrm{r} \text {,neo }}$ during all phases of the LCO. Within the error bars and time resolution of the system $(100 \mu \mathrm{s})$, we therefore could not find any indication of net flows beyond the neoclassical expectations (e.g. low frequency zonal flows).

The modulation of the density profile is also illustrated in figure 2, which shows the spatio-temporal correlation of the $f_{D \text {-signal }}\left(\sim u_{\perp}\right)$ of the Doppler reflectometer $\left(\rho_{\text {pol }} \approx 0.995\right)$ with different radial channels of the raw data from Li-BES (time resolution $5 \mu \mathrm{s}$ ). The Li-BES raw data is in the edge of the plasma a proxy for the electron density (see the discussion of the limitation of this 


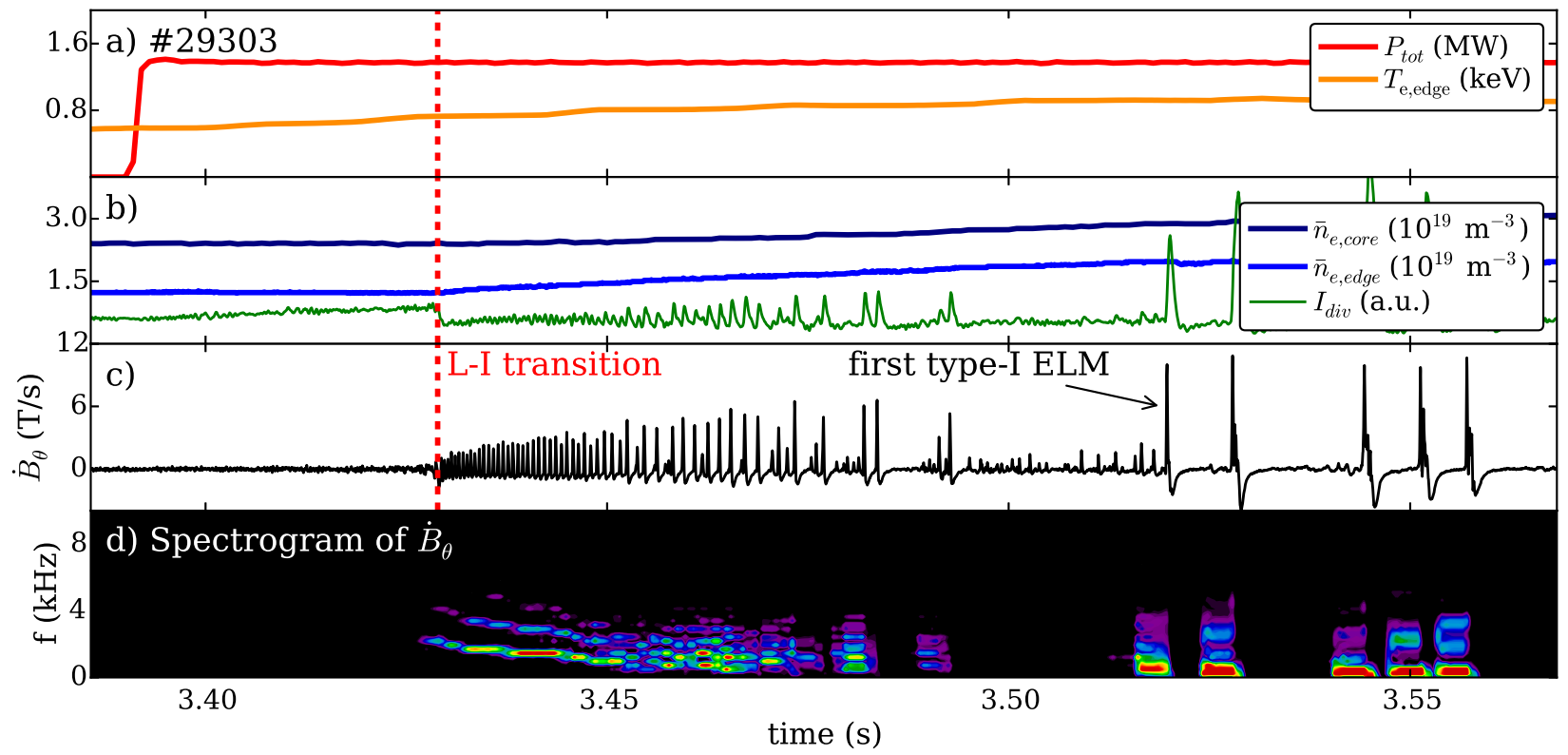

FIG. 3: (a) Heating power (red) and edge electron temperature (orange), (b) line averaged density (dark blue: core, light blue: edge) and divertor shunt current (green) for a neutral beam heated L-H transition. The LCOs are clearly visible in the signal of the poloidal magnetic field pick-up coil below the divertor (c). Its spectrogram (d) exhibits higher harmonics at the beginning which vanish due to increasing degree of intermittency of the LCOs in the later phase.

interpretation in reference [42]). The correlation of the reference signal $x_{\text {ref }}$ (from Doppler reflectometry) with the signal $x_{i}$ (from Li-BES channel $i$ ) is calculated as the normalised cross-correlation function

$$
C_{i}(\Delta t)=\frac{\left\langle x_{\mathrm{ref}}(t) x_{i}(t+\Delta t)\right\rangle}{\sigma_{\mathrm{ref}} \sigma_{i}}
$$

with time delay $\Delta t$ and the standard deviations $\sigma_{\text {ref }}$ and $\sigma_{i}$ of the reference signal and the signal of channel $i$ under consideration, respectively. $\langle\cdot\rangle$ represents a temporal average (in the considered case from $t=3.465$ to 3.475 $\mathrm{s})$. The different cross-correlation functions are placed to the radial position of the line of sight of the considered Li-BES channel $i$ given in the normalised poloidal flux coordinate $\rho_{\text {pol }}$.

The $2 \mathrm{kHz}$ modulation of the cross-correlation function is strongest (green cross) at a radial position of $\rho_{\text {pol }}=0.99$ (correlation up to 0.2 ) and propagates from there radially outward into the far scrape-off layer with a velocity of about $v_{r} \approx 100-300 \mathrm{~m} / \mathrm{s}$. This can be deduced from the regular blue (negative correlation) and red (positive correlation) tilted stripes which extend from $\rho_{\text {pol }}=0.98$ to 1.07. Whether the modulation of the density takes place likewise further inside than $\rho_{\text {pol }}=0.98$ cannot be evaluated since the sensitivity of the Li-BES system is decreased in that region [42]. In this sense, it is possible that the LCO modulation of the density in fact exists in a wider radial region than indicated by figure 2 . As reported in reference [3], the strongest modulation of the perpendicular flow velocity was found slightly in- side the separatrix, and the flow profile pulsates in a radial range from $\rho_{\text {pol }}=0.98$ into SOL which agrees with the radial range of the modulation in the Li-BES signals. However, a radial propagation as indicated by phase shifts in the Li-BES data was not found in the Doppler data. The radial propagation of the density perturbation might explain the anti-correlation between the Doppler signals and the ion-saturation current signal from a divertor X-point probe as reported in reference [4].

If the heating power is chosen marginally close to the L-H power threshold, the I-phase can be kept stable for a whole discharge. For slightly higher heating power, the temperature and density will rise until the first type-I ELM appears [43]. Also in this case an I-phase is observed, and the frequency of the LCOs decreases steadily sometimes followed by an ELM-free H-mode phase. An example of such a discharge $\left(\# 29303, B_{t}=-1.8 \mathrm{~T}\right.$, $I_{p}=0.6 \mathrm{MA}$ ) is illustrated in figure 3. After switching on the neutral beam injection (NBI), the temperature rises continuously (a). With the drop of the divertor current (green line in (b)) at $t=3.429 \mathrm{~s}$, also the density (blue) starts to rise and the LCOs are clearly visible in the signal $\dot{B}_{\theta}$ of the poloidal magnetic field pick-up coil below the divertor (c). As seen in the spectrogram of the $\dot{B}_{\theta}$-signal (d), the frequency slightly decreases with increasing density, and in the early I-phase, higher harmonics can clearly be identified. These harmonics are a result of the non-sinusoidal shape of the LCO pulses and are a unique characteristic in the $\dot{B}_{\theta}$-signal which allows to identify LCOs and (closeness to) L- to H-transitions in 


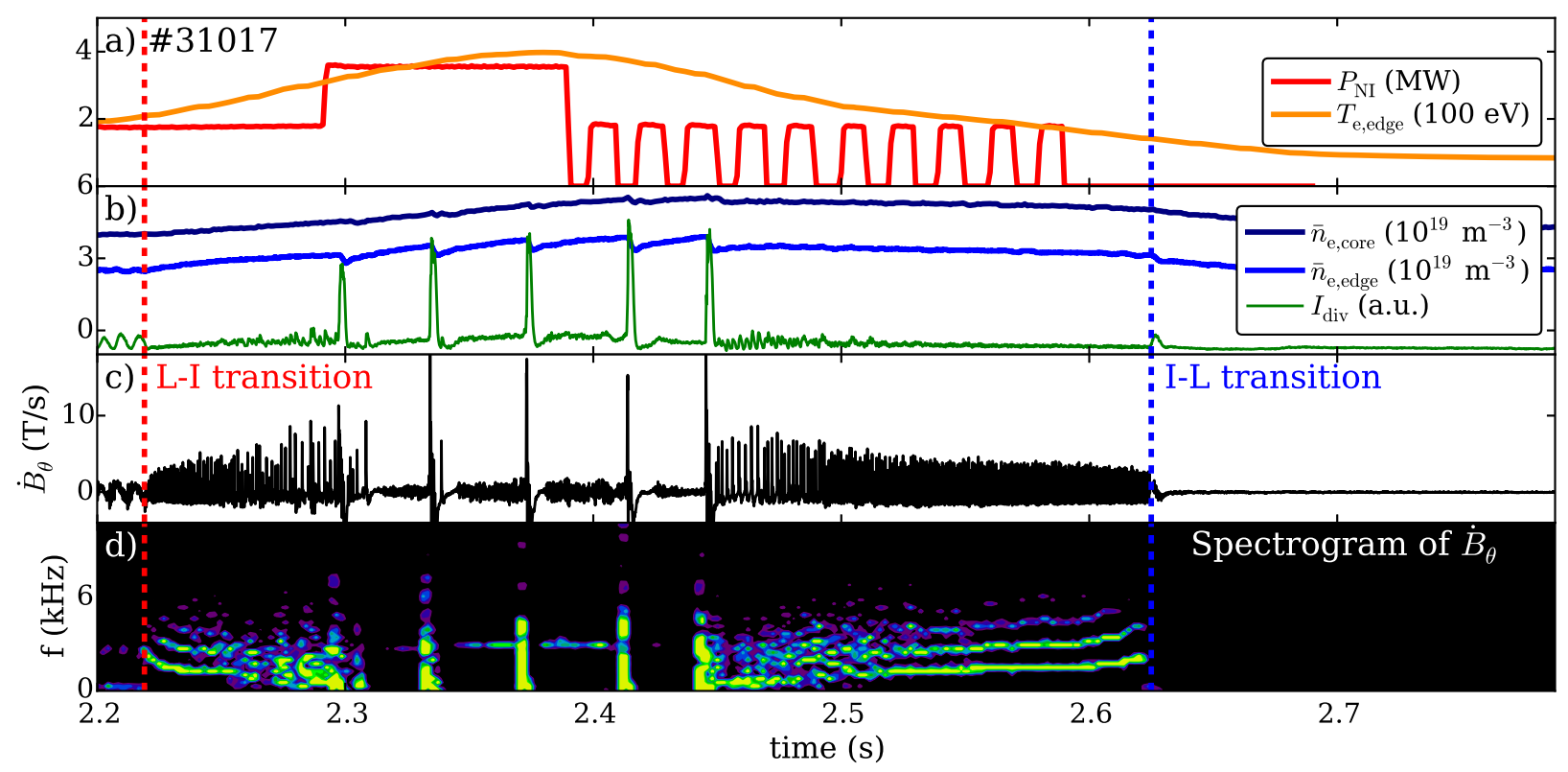

FIG. 4: Example of an L- to H-transition (red dashed line) induced by injection of neutral beam power and back transition (blue dashed line) to L-mode after a short $\mathrm{H}$-mode phase in the same representation as figure 3. LCOs appear at the L-H transition as well as at the back transition to L-mode as recognisable by the higher harmonics in the spectrogram of the magnetic signal.

a convenient way. After some time, the higher harmonics disappear in the spectrogram as a consequence of the irregular occurrence of the LCO pulses. Obviously, the I-phase transitions smoothly from a very regular state into an intermittent state. Bursts as seen in the intermittent phase of the LCOs are also found between type-I ELMs, and in very fast heating ramps only this bursty type of LCO spikes appear. In addition, they are similar to bursts which have recently been found in Doppler reflectometry data of I-mode discharges (electron $\mathbf{B} \times \boldsymbol{\nabla} B$ drift towards X-point ) at AUG [44].

\section{LCOS AT THE TRANSITION FROM H- TO L-MODES}

The dynamics of the I-phase at the back transition from $\mathrm{H}$ - to L-mode (H-L transition) is reversed in comparison to the L-H transition (forward transition). This is illustrated in figure 4, which shows the evolution from L-mode to a short phase of an ELMy H-mode and back to L-mode. Similar to the case described in figure 3, the LCOs in this discharge (\#31017, $B_{t}=-3.0 \mathrm{~T}, I_{p}=$ $0.8 \mathrm{MA}$ ) start very regular at $t=2.219 \mathrm{~s}$ after switching on the NBI. Afterwards, they decrease in frequency, and are getting intermittent later until a type-I ELM appears as visible as a large spike in the divertor current (green) in figure 4(b). On the way back to L-mode after four additional type-I ELMs, the LCOs are initially quite intermittent starting directly after the last type-I ELM crash at $t=2.45 \mathrm{~s}$. The frequency is getting more and
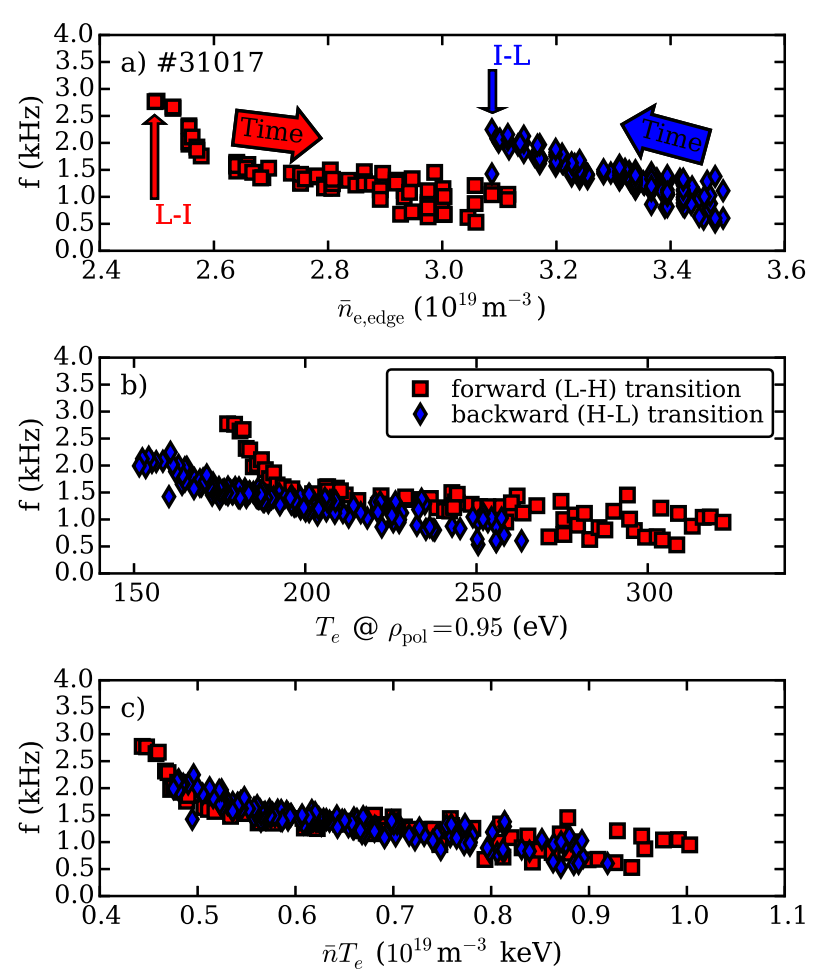

FIG. 5: LCO frequency dependence on density (a), temperature (b) and pressure (c) for the discharge shown in figure 4. The density is lower in the LCO state at the L-H transition (squares) and the temperature higher. For the I-phase at the $\mathrm{H}-\mathrm{L}$ transition (diamonds) the opposite is the case. 


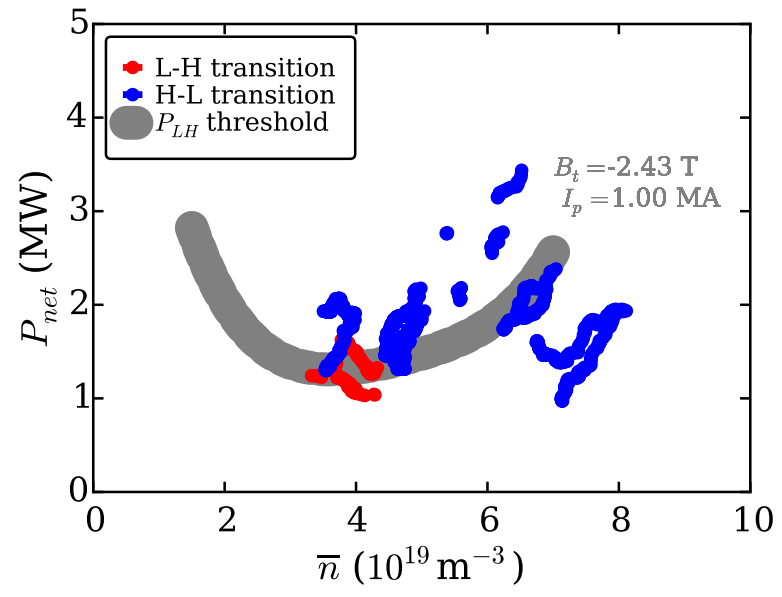

FIG. 6: Existence of LCOs depending on net heating power $P_{\text {net }}$ and line averaged core density $\bar{n}$ for a set of comparable discharges with $B_{t}=-2.43 \mathrm{~T}$ and $I_{p}=1 \mathrm{MA}$. LCOs at the transition from $\mathrm{H}$ - to L-mode (blue) are found at higher densities relative to the LCOs at the L- to H-transition (red). The grey bar indicates the L-H power threshold as presented in reference [47].

more stable, and higher harmonics appear in the spectrogram of the $\dot{B}_{\theta}$-signal (figure $4(\mathrm{~d})$ ). The frequency slowly increases simultaneously to the decrease of density and temperature, and stays almost fixed in the last phase of the LCO for about $100 \mathrm{~ms}$. When the LCOs stop at $t=2.625 \mathrm{~s}$, the edge density drops faster and the divertor shunt current is transiently increased which indicates a drop in confinement.

Due to better particle confinement in H-mode, the density during the I-phase at the back transition from $\mathrm{H}$ - to L-mode is higher than at the forward transition which typically starts from lower L-mode densities. This is illustrated in figure 5(a) which shows the density dependence of the LCO frequency at the forward (squares) and backward (diamonds) transition. In both cases the LCO frequency depends almost linearly on the line averaged edge density $\bar{n}_{\mathrm{e}, \text { edge }}$.

As shown in figure $5(\mathrm{~b})$, the frequency also shows a monotonic dependence on the electron temperature at a radial position of $\rho_{\text {pol }}=0.95$. Here, the electron temperatures are taken from the integrated data analysis (IDA) shotfiles [45], and are mainly determined by the ECE data in this radial region. The temperatures are in general higher at the the beginning of the I-phase at the forward transition in comparison to the end of the LCO state at the backward transition. This is due to the fact that the heating is already switched on at the forward transition in order to push the L-mode into the LCO state, while the heating is already switched off at the backward transition.

The electron pressure, estimated as the product $p=$ $\bar{n}_{\mathrm{e}, \text { edge }} T_{e}$ seems to be identical in equivalent phases of the LCOs at the forward and at the backward transition (figure 5(c)). This was already found for the pressure directly at the forward and back transitions to and from H-mode [43]. The onset and the frequency of the LCOs seem therefore to be independent of collisionality since it is quite different at the forward and back transition.

Figure 5(c) indicates a decreasing pressure dependence of the LCO frequency. However, it is not clear whether it is really a pressure dependence or a dependence on the pressure gradient which is related to the neoclassical radial electric field and therefore perpendicular $E \times B$-flow. A frequency scaling depending on $p$ could also indicate a dependence on the normalised pressure $\beta$ (plasma beta) which is a key quantity determining the stability of MHD modes and microinstabilities [46]. We will discuss this issue in Section VI when a larger data set is considered.

The fact that the density during the I-phase is higher at the back transition from $\mathrm{H}$ - to L-mode extends the range of existence of the I-phase significantly. In contrast to the existence plot of the I-phase as shown in figure 1 (a) of reference [3], the accessible density range extends to much higher densities up to $\bar{n}_{\mathrm{e}, \mathrm{core}}=8 \cdot 10^{19}$ $\mathrm{m}^{-3}$ if the backward transitions are taken into account. This is illustrated in figure 6 which shows the I-phases at forward (red) and backward (blue) transitions in the power-density plane for a set of $\mathrm{L}-\mathrm{H}$ and $\mathrm{H}-\mathrm{L}$ transitions at fixed $B_{t}=-2.43 \mathrm{~T}$ and $I_{p}=1 \mathrm{MA}$ from discharges \#27124, \#27126 and \#27129. The net power $P_{\text {net }}=P_{\text {heat }}-d W_{\mathrm{MHD}} / d t$ is the heating power $P_{\text {heat }}$ taking into account losses and absorption coefficients of the different heating methods, and $W_{\mathrm{MHD}}$ is the plasma energy from the equilibrium. While the I-phases at the forward transition are typically found below $\bar{n}_{\mathrm{e}, \text { core }}=5 \cdot 10^{19}$ $\mathrm{m}^{-3}$ and very close to the L-H power threshold $P_{\mathrm{LH}}$ (grey bar) [47], they are widely spread in terms of density at the backward transition. The scatter of the latter in terms of $P_{\text {net }}$ is mainly attributed to the radiative power loss which is not taken into account for the estimation of $P_{\text {net }}$ and therefore introduces large inaccuracies. However, this does not affect the conclusions about the extended density range which is accessible for the I-phases at the $\mathrm{H}-\mathrm{L}$ transitions.

\section{MAGNETIC STRUCTURE OF LCOS}

As already presented in Section II and III, there is a magnetic activity detectable in the poloidal magnetic field pick-up coil located below the divertor during the I-phase. This is, however, not the only coil which shows clearly the LCO pulsation. At AUG, a poloidal array of $\dot{B}_{\theta}$-probes exists as shown in figure 7 (b) covering the whole circumference. The time traces from some of these coils during two LCO pulses of discharge \#29302 (cf. figure 1) are depicted in figure $7(\mathrm{a})$. The signals below the divertor (probes 22 to 25 ) exhibit the highest pulse amplitude, but the LCOs are also clearly visible on probes along the high field side (HFS) up to the top (probes 6 to 

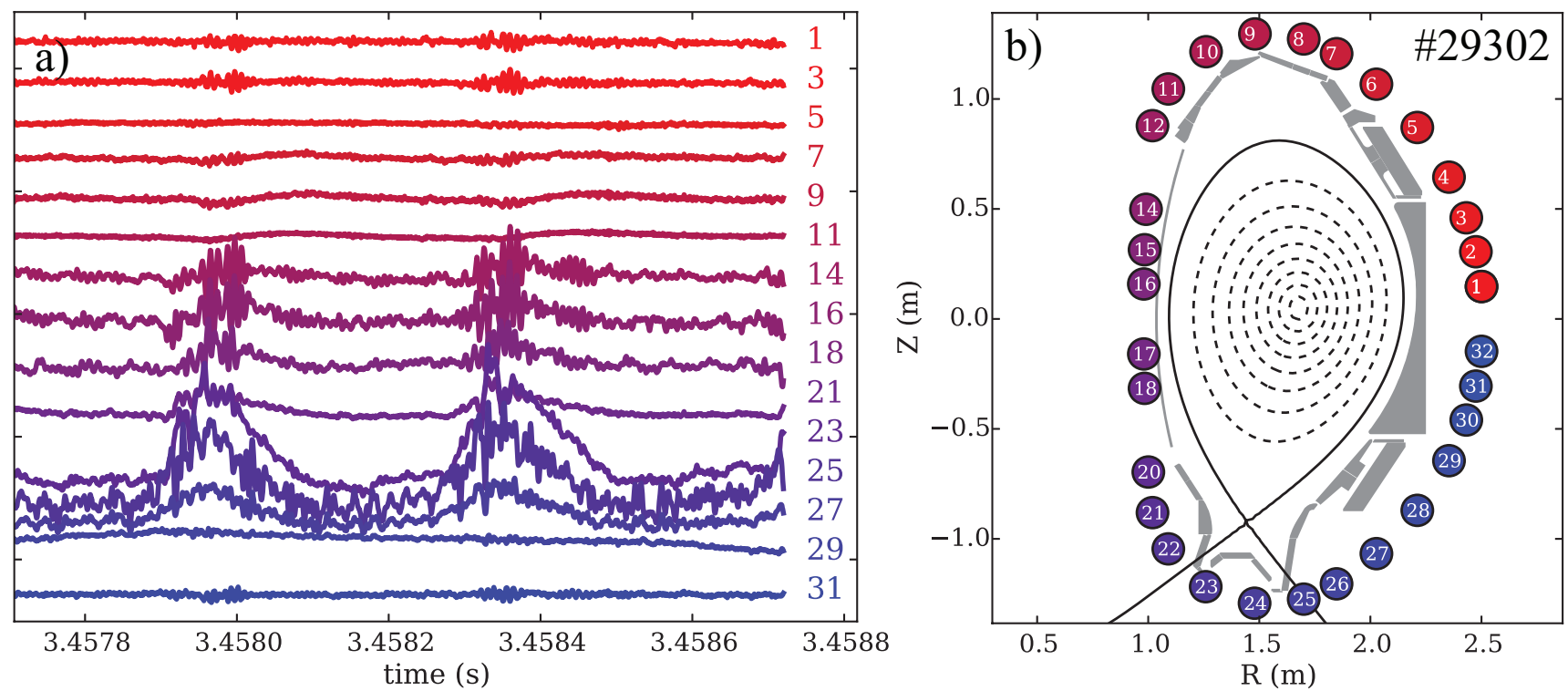

FIG. 7: Selected signals (a) of magnetic pick-up coils measuring $\dot{B}_{\theta}$ from a poloidal probe array (b) for discharge \#29302 (cf. figure 1) during the I-phase. The strongest pulsation is found in probes below the divertor (probes 22 to 25 ).

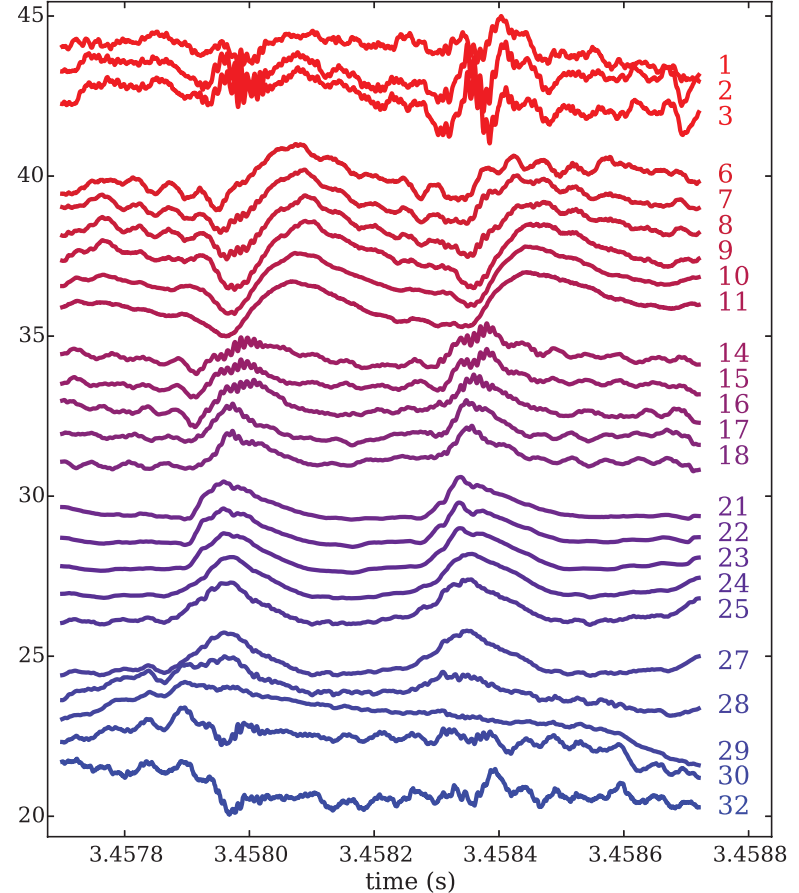

FIG. 8: Same signals and probe numbering as shown in figure 7 (a) but normalised to the maximum value of the respective signal. The propagation from bottom to top is clearly visible.

28). At the midplane low field side (LFS), the magnetic probes do not show clear pulsations which is possibly due to the large distance of the probes from the plasma or due to interference with structure material and the associated decreasing sensitivity of the magnetic pick-up coils.

The signals detected at the top of the plasma (e.g. with probe 9) during a LCO gives rise to higher harmonics in the spectrogram (not shown) similar to the case shown in figure 3. There is, however, a time delay between the pulse at the bottom (e.g. probe 23) and the top (e.g. probe 9 ). This delay is visible in figure 8 which shows the same signals as in figure $7(\mathrm{a})$ but normalised to the maximum of the respective time trace and filtered by a low-pass filter with a limit frequency of $200 \mathrm{kHz}$. The pulsations start from the bottom and propagate along the HFS to the top almost keeping the waveform except for the signals from the LFS (probes 1 to 5 and probes 29 to 32$)$.

The propagation and mode structure can be analysed in more detail by applying a cross-correlation analysis on all the probe signals. For this purpose, we choose probe 25 as a reference and cross-correlate this signal $x_{\text {ref }}(t)$ with all other $\dot{B}_{\theta}$-probe signals $x_{i}(t)$ evaluating the normalised cross-correlation function as given by equation 1 . The result is shown in figure 9(a). At the LFS around $\theta=0.0$, the correlation is very low while the highest values are achieved around the reference probe (green symbol, $\theta \approx-\pi / 2)$, at the high field side $(\theta= \pm \pi)$ and at the top of the plasma $(\theta \approx \pi / 2)$. The tilt of the regions of high correlation (red) indicates a propagation from the bottom to the top as already seen figure 8 . From the cross-correlation function we use the correlation at time delay $\Delta t=0, C_{i}(0)$, in order to study the poloidal structure of the LCO pulsations. As indicated in figure $9(\mathrm{~b})$, the highest correlation $C_{i}(0)$ with respect to the reference probe (probe 25) is found in the signals of the bottom probes located in the vicinity of the reference probe. The correlation reverses sign in the upper 

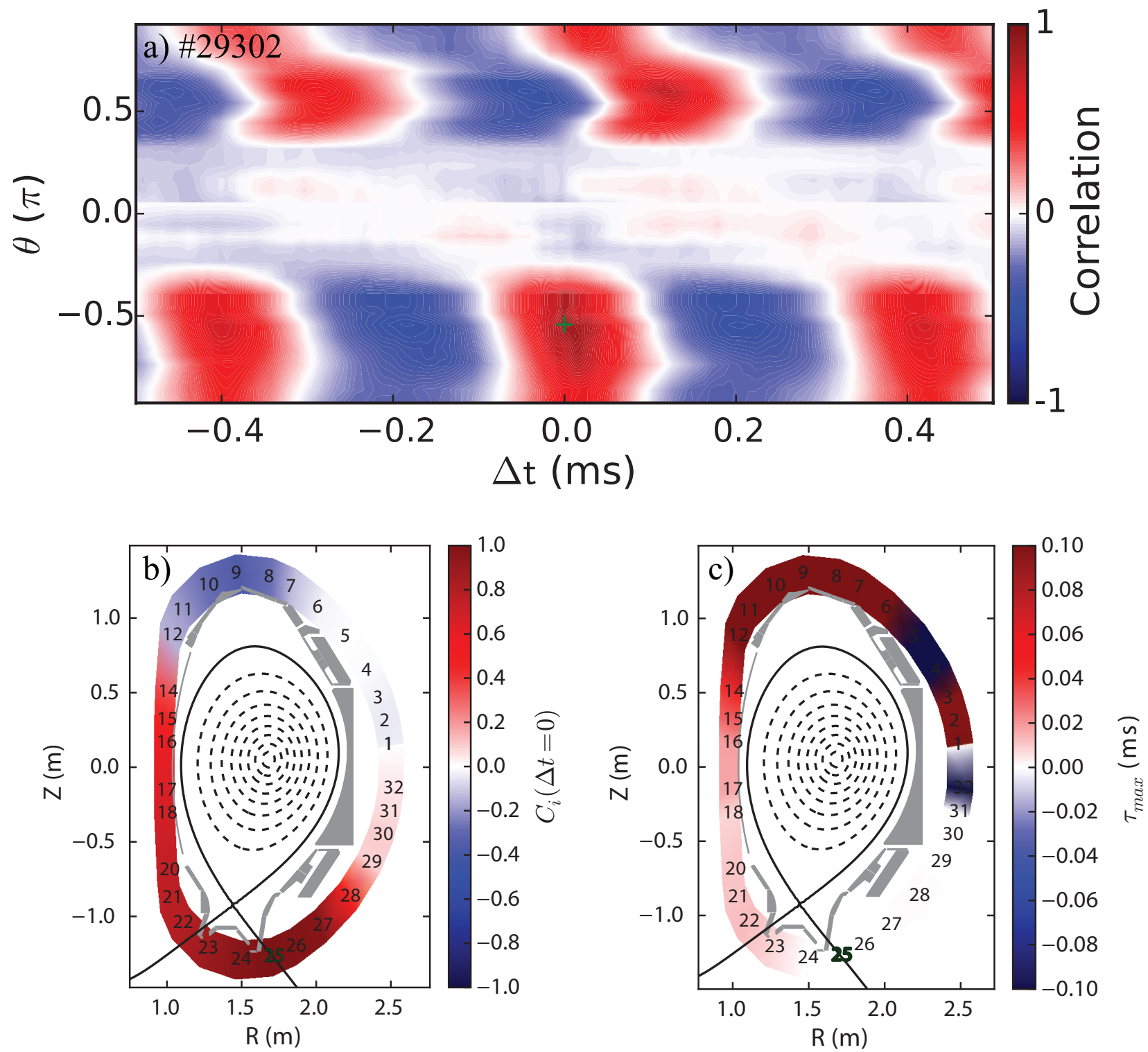

FIG. 9: Poloidal mode structure of $\dot{B}_{\theta}$-signals from cross-correlation analysis (a) with reference probe 25 (green). The correlation at time delay $\Delta t=0(\mathrm{~b})$ reveals the up-down asymmetry of the LCO pulsation. The pulsation starts at the reference probe and propagates upwards along the HFS resulting in increasing time delays $\tau_{\max }$ (c).

half of the plasma and reaches maximum anti-correlation $C_{i}(0)=-0.35$ at probe 9 at the very top of the plasma. This reveals the up-down asymmetry of the poloidal magnetic field perturbation at the peak of the LCO pulses.

The time delay $\tau_{\max }$, at which the cross-correlation function is maximum, is used to determine the time lag of the pulsations with respect to the reference probe. The result is shown in figure $9(\mathrm{c})$. The time lag $\tau_{\max }$ is zero at the reference probe (due to the properties of the auto-correlation function) and increases continuously from probe to probe along the HFS up to probe 9, which has the maximum time lag of $\tau_{\max }=124 \mu \mathrm{s}$ with respect to the reference probe. This corresponds to a propagation velocity of the LCO pulse of $v_{\text {pulse }}=L_{\|} / \tau_{\max } \approx 91 \mathrm{~km} / \mathrm{s}$ from bottom to top (or the ion diamagnetic direction in the lab frame) if we approximate the parallel connection length of the field line with $L_{\|} \approx q_{95} R_{0}=11.3 \mathrm{~m}$ with edge safety factor $q_{95}$ and major plasma radius $R_{0}$. This agrees well with the sound velocity $c_{s}=\sqrt{T_{e} / m_{i}}$ in the edge plasma ( $m_{i}$ is the ion mass).

In upper single null discharges with the X-point at the top and reversed toroidal field (this corresponds again to favourable ion $\mathbf{B} \times \nabla B$-drift), we observe the same dynamics but reversed. This means, the maximum 

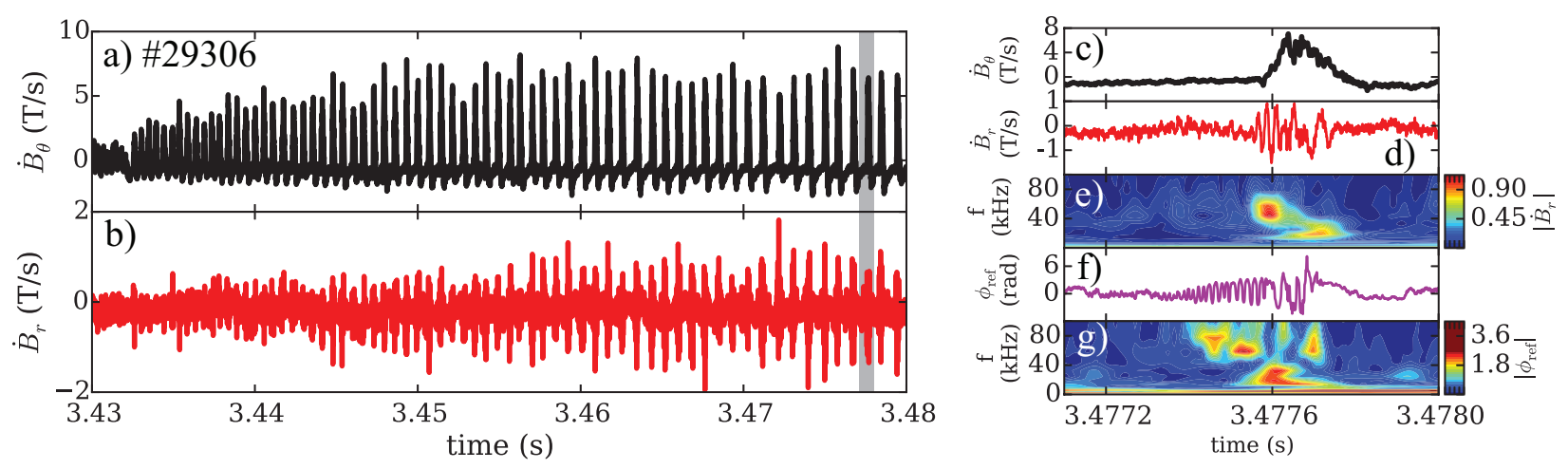

FIG. 10: Poloidal (a) and radial (b) magnetic field signals from pick-up coils during a neutral beam heated I-phase (\#29306). The late I-phase (shaded area in (a)) is shown more detailed in (c)-(g). Prior to the poloidal magnetic field perturbation (c), precursor activity is found in the raw signal (d) and wavelet spectrum (e) of $\dot{B}_{r}$. This precursor is found even earlier in the phase signal $\phi_{\text {ref }}$ of the hopping reflectometer (f) and shows activity in the frequency range 50 to $100 \mathrm{kHz}$ as seen in its wavelet spectrum (g).

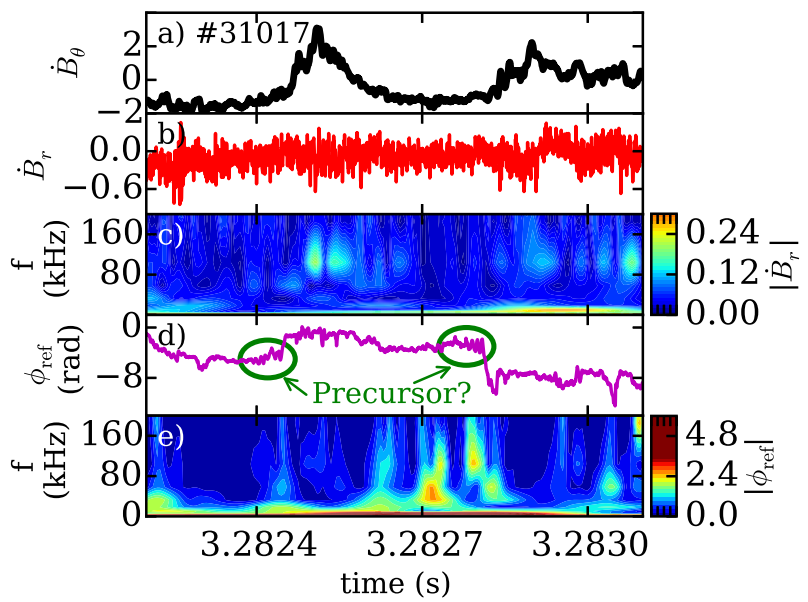

FIG. 11: The very first two LCO pulses (early LCO) from discharge \#31017: poloidal (a) and radial (b) magnetic field signals from pick-up coils, and wavelet spectrum of the $\dot{B}_{\theta^{-}}$ signal (c). The phase signal $\phi_{\text {ref }}$ of the hopping reflectometer (d) and its wavelet spectrum (e) indicate a weak precursor activity.

amplitude of the $\dot{B}_{\theta}$-signal is found at the top of the plasma, and the pulse propagates along the HFS downwards (which is again the ion diamagnetic direction in the lab frame). As already mentioned, we do not observe these regular LCOs in unfavourable configuration (electron $\mathbf{B} \times \nabla B$-drift towards X-point), and can therefore not perform the correlation analysis in the same way for these cases.

Thus, we observe in general a strong poloidal magnetic field perturbation during the I-phase starting from the active divertor $\mathrm{X}$-point and propagating into the ion diamagnetic direction along the HFS.

\section{PRECURSOR ACTIVITY DURING LCOS}

While the poloidal magnetic pick-up coils register strong perturbations of the poloidal magnetic field $B_{\theta}$ during LCOs as decribed before, the radial magnetic field perturbations $\dot{B}_{r}$ measured with pick-up coils at the LFS are much weaker. This is illustrated in figure 10 . The poloidal magnetic field signal (a) is already strong (up to $5 \mathrm{~T} / \mathrm{s})$ at the beginning of the $\operatorname{LCO}(t=3.432 \mathrm{~s})$ while the radial magnetic field signal (b) is hardly above the noise level $(\approx 0.2 \mathrm{~T} / \mathrm{s})$. In the following evolution, however, both signals increase and in the late I-phase $(t>3.46 \mathrm{~s})$ the $\dot{B}_{r}$ exhibits clearly visible spikes appearing with LCO frequency.

A zoom into the late I-phase (figure $10(\mathrm{c})-(\mathrm{g})$ ) reveals that the magnetic signature of the $\dot{B}_{r}$-signal (d) differs significantly from the $\dot{B}_{\theta}$-signal (c). While a LCO pulse appears as a single peak in the $\dot{B}_{\theta}$-signal, the $\dot{B}_{r}$-signal features a short oscillation starting prior to the $\dot{B}_{\theta}$-pulse. The frequency of this precursor oscillation as deduced from the wavelet spectrum of the $\dot{B}_{r}$-signal (e) is 40 to $70 \mathrm{kHz}$. We use here a Morlet wavelet for the continuous wavelet transform (for details of the wavelet transform see reference [48]).

Even before the oscillation is seen in the $\dot{B}_{r}$-signal, it can be detected with the LFS hopping reflectometer [49]. The probing frequency in the considered time range was set to $33 \mathrm{GHz}$ corresponding to a cutoff density of $1.35 \cdot 10^{19} \mathrm{~m}^{-3}$ which is in the considered case close to the LCFS $\left(\rho_{\text {pol }}=1.0 \pm 0.01\right)$. The phase signal $\phi_{\text {ref }}$ of this diagnostic is for low density perturbations proportional to the radial position of the cutoff-density layer. Due to this, the oscillation as seen in figure 10 (f) from $t=3.4773$ to 
3.4776 s can be interpreted as an oscillation of the cutofflayer caused by a regular modulation of the electron density. However, a misalignment of the antennas could likewise result in a phase modulation caused by propagating density perturbations. In any case, the precursor mode whether being a density or a velocity modulation-starts at $t=3.4773 \mathrm{~s}$ with a frequency of about $80 \mathrm{kHz}$ as seen in the wavelet spectrum (g), increases in amplitude, and disappears when the LCO pulse has reached the maximum in the $\dot{B}_{\theta}$-signal. The reflectometer phase signal jumps usually up or down ('runaway phase' due to large amplitudes of the density perturbations, finite beam width and angle of incidence of the probing microwave [50]) at the maximum of the LCO pulse as it is typical during an ELM crash or other large transient events [51] indicating a strong density perturbation during the LCO pulse. From the poloidal correlation reflectometer system [52] a perpendicular wavenumber of the precursor oscillation is estimated to be $k_{\perp}=0.28 \mathrm{~cm}^{-1}$. This corresponds approximately to a poloidal modenumber of $m \approx 15$. The poloidal velocity of the precursor is about $12 \mathrm{~km} / \mathrm{s}$ and points into the electron diamagnetic direction with respect to the lab frame. This indicates that the precursor modes are localised close to the $E_{r}$-minimum in agreement with the fact that they can only be detected with the reflectometer very close to the separatrix.

These observations suggest that there is a growing precursor mode which couples non-linearly to other modes, when a sufficiently high amplitude is reached, finally triggering an ejection of particles [53]. At sufficiently high amplitude, this mode is accompanied by electromagnetic activity which can be detected with the radial magnetic pick-up coil. This dynamics together with the measured frequency range and the poloidal size is in agreement with the definition of type-III ELM precursors [54].

The existence of type-III ELM precursors in the late I-phase was already reported from other fusion experiments [5-8]. The precursors, however, have not been clearly observed at the beginning of the I-phase in the magnetic signal $\dot{B}_{r}$. As shown in figure 11 for the two very first LCO pulses from discharge \#31017 $\left(B_{t}=-3.0\right.$ $\left.\mathrm{T}, I_{p}=0.8 \mathrm{MA}\right)$, precursor activity is visible neither in the radial magnetic field signal (b) nor its wavelet spectrum (c) prior to the LCO pulses.

However, the phase signal of the reflectometer (figure $11(\mathrm{~d})$ ) systematically exhibits small oscillations prior to the LCO pulse followed by a runaway phase as described above. Although these precursor oscillations are weak in the early I-phase, they are found systematically before most of the LCO pulses, if the probing frequency of the microwave corresponds to a radial position close to the LCFS. As seen in the wavelet spectrum of the reflectometer phase figure 11(e) prior to the second LCO pulse $(t=3.2828 \mathrm{~s})$, the frequency of the weak precursors in the first LCO pulses is higher (typically $f \approx 100$ to 200 $\mathrm{kHz})$ than in the late I-phase $(f \approx 50$ to $100 \mathrm{kHz}$, see figure $10(\mathrm{~g}))$.

\section{FREQUENCY SCALING OF LCOS}

As shown in figures 3,4 and 5, the LCO frequency decreases with increasing density and temperature, and therefore pressure. In order to investigate the frequency dependence on further parameters like the toroidal magnetic field $B_{t}$ and the plasma current $I_{p}$, we choose a set of discharges with comparable plasma shape but varying $B_{t}$ and $I_{p}$. The discharges are heated with neutral beam injection launched in a single power step from 0 to $P_{\mathrm{NBI}}$ marginally above the L-H power threshold. Examples of this type of discharges are given in figures 1 and 3 , and further details are summarised in table I.

\begin{tabular}{|c|c|c|c|c|c|}
\hline discharge number & $B_{t}(\mathrm{~T})$ & $I_{p}(\mathrm{MA})$ & $q_{95}$ & $f_{G}$ & $P_{\text {NBI }}(\mathrm{MW})$ \\
\hline$\# 29302$ & -2.5 & 0.6 & 6.85 & 0.35 & 1.4 \\
$\# 29303$ & -1.8 & 0.6 & 4.96 & 0.35 & 1.4 \\
$\# 29306$ & -1.4 & 0.6 & 3.85 & 0.37 & 0.9 \\
$\# 29307$ & -1.4 & 0.6 & 3.85 & 0.35 & 0.9 \\
$\# 29308$ & -3.2 & 0.6 & 8.72 & 0.35 & 1.7 \\
$\# 29309$ & -2.5 & 0.83 & 4.90 & 0.25 & 1.7 \\
$\# 29310$ & -1.4 & 0.47 & 5.00 & 0.45 & 0.9 \\
$\# 29311$ & -1.8 & 0.6 & 4.94 & 0.35 & 0.9 \\
$\# 29312$ & -1.8 & 0.6 & 4.96 & 0.36 & 0.9 \\
$\# 29315$ & -3.2 & 1.07 & 4.96 & 0.22 & 2.1 \\
\hline
\end{tabular}

TABLE I: Discharge number, toroidal magnetic field $B_{t}$, plasma current $I_{p}$, edge safety factor $q_{95}$, Greenwald fraction $f_{G}$ and neutral beam heating power $P_{\mathrm{NBI}}$ used for the frequency scaling experiments.

By means of this set of discharges, we study the dependence of the LCO frequency during the first $60 \mathrm{LCO}$ cycles on the edge density (approximated by the line averaged edge density $\bar{n}_{\text {e,edge }}$ ), electron temperature $T_{e}$ measured with Thomson scattering [55] at $\rho_{\text {pol }}=0.95$, toroidal magnetic field $B_{t}$, toroidal plasma current $I_{p}$, and edge safety factor $q_{95}$.

First, we compare the measured LCO frequency with a prediction of the LCO frequency derived from a model based on mean field momentum transport equations [34]. In this model, the frequency of the LCO increases approximately linearly with the neoclassical poloidal damping rate $\nu_{\text {neo,damp }}$ if all model parameters (except for the density entering $\nu_{\text {neo,damp }}$ ) are kept fix. Since the edge parameters of the considered discharges are in the transition region between banana and plateau regime, the multi-regime approximation of the damping rate [56]

$$
\nu_{\text {neo,damp }}=\frac{g \hat{\mu}_{00}^{B} \nu_{i i}}{\left(1+2.92 \nu^{*} \hat{\mu}_{00}^{B} / \hat{\mu}_{00}^{P S}\right)\left[1+\hat{\mu}_{00}^{P} \nu_{i i} /\left(6 \omega_{t} \hat{\mu}^{P S}\right]\right.}
$$

is used. It depends on tabulated dimensionless viscosities $\hat{\mu}_{00}$ in the banana (B), plateau (P) and Pfirsch-Schlüter (PS) regime [56], on the ion-ion collision frequency $\nu_{\mathrm{ii}}$, on the ion transit frequency $\omega_{t}=\sqrt{T_{i} / m_{i}} /\left(R_{0} q_{95}\right)$, and 

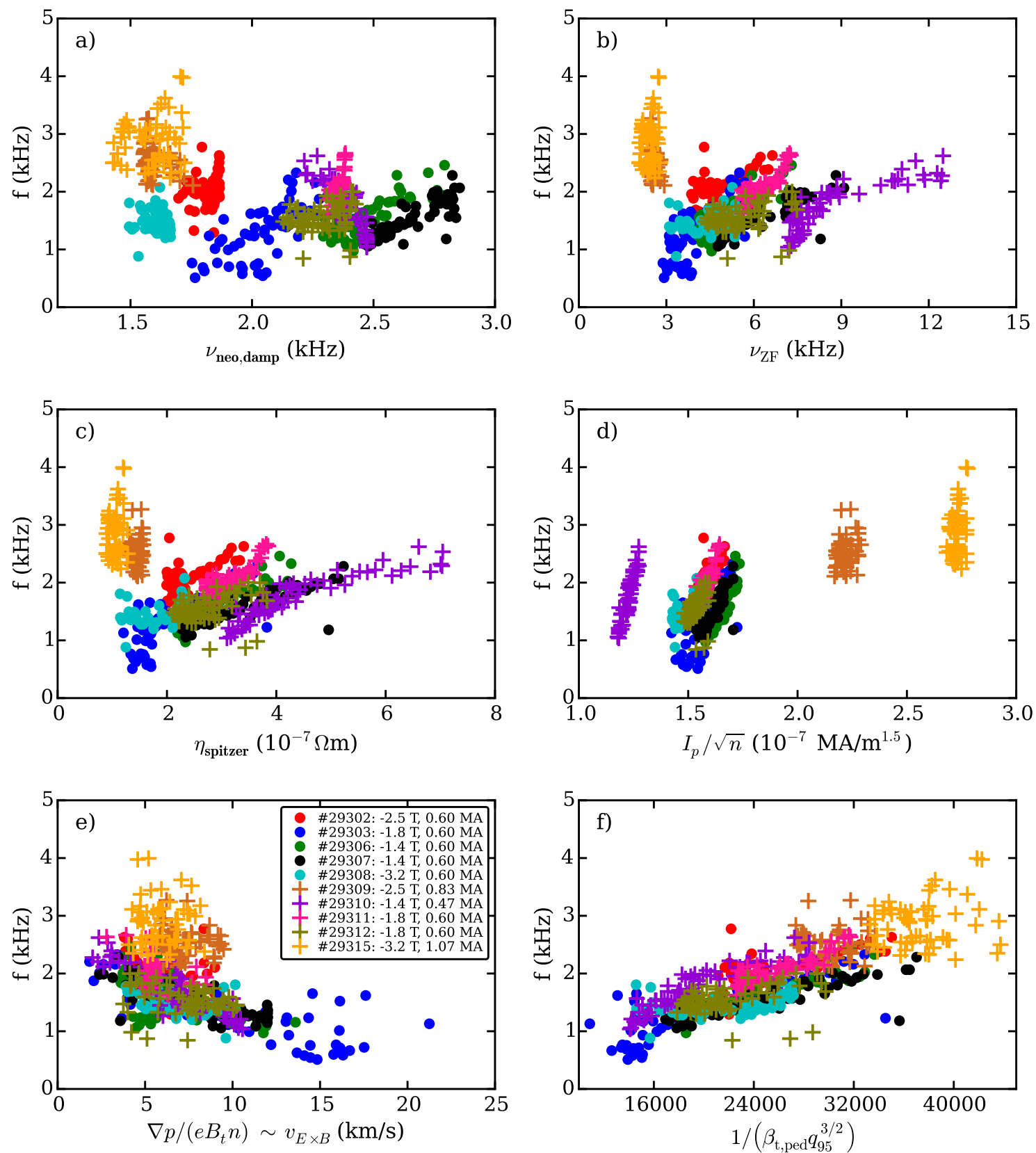

FIG. 12: Dependence of the LCO frequency $f$ on the neoclassical poloidal damping rate (a), zonal flow damping rate (b), Spitzer resistivity (c), the JET M-mode scaling formula (d), estimated $E \times B$-flow velocity (e), and a fit formula based on a linear regression (f) for a set of discharges. Frequencies from individual discharges are depicted in the same color.

on the normalised ion collisionality [57]

$$
\nu^{*}=4.9 \cdot 10^{-18} \frac{q_{95} R_{0} n_{i} \ln \Lambda_{i i}}{T_{i}^{2} \epsilon^{3 / 2}}
$$

$\epsilon$ is the local inverse aspect ratio, $R_{0}$ the major plasma radius, and $\ln \Lambda_{i i}=30-\ln \left(\sqrt{n_{i}} / T_{i}^{3 / 2}\right)[57]$ the Coulomb logarithm of deuterium ions. The factor $g$ can be approx- imated with high accuracy [56] by

$$
g=\frac{1}{1-1.46 \sqrt{\epsilon}+0.46 \epsilon \sqrt{\epsilon}}-1 .
$$

As shown in figure 12(a), the absolute values of the neoclassical poloidal damping rate $\nu_{\text {neo,damp }}$ are in the same order of magnitude as the measured LCO frequency which is an important prerequisite to play a role in the dynamics of a LCO. In addition, the LCO frequencies of 
some individual discharges (one color corresponds to the covered LCO frequency range of one discharge) seem to increase with $\nu_{\text {neo,damp }}$ (e.g. \#29303, \#29306, \#29307) as expected. However, other discharges do only weakly depend on the estimated poloidal damping rate and the overall picture does not confirm a linear frequency dependence on $\nu_{\text {neo,damp. }}$ For the evaluation of $\nu_{\text {neo,damp, we }}$ used $T_{i}=T_{e}$ due to the lack of accurate ion temperature measurements in the edge plasma. This could be a source of errors, and, in addition, we neglected any dependence of the flux surface geometry, $q_{95}$ or inertia which could affect the frequency of the LCO model presented in reference [34]. For a quantitative comparison a full kinetic

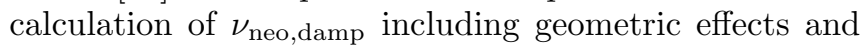
a more elaborate comparison with the equation system of the model has to be done instead which is out of the scope of this Letter.

Following the LCO model based on a zonal flowturbulence interaction [29, 31], the LCO frequency should depend on the collisional damping of the zonal flow if turbulent self-damping by non-linear saturation is weak [58] as can be expected for the I-phase which is a regime of reduced turbulence intensity. As described in reference [59], the zonal flows can be damped in several ways. Despite the different damping processes, the dominant collisional damping rate of the zonal flow can be estimated as

$$
\nu_{\mathrm{ZF}}=\nu_{\mathrm{ii}} /(1.5 \epsilon)
$$

with the inverse aspect ratio $\epsilon$ and the ion-ion collision frequency $\nu_{\mathrm{ii}}$. A large damping rate $\nu_{\mathrm{ZF}}$ would correspond to a large zonal flow damping and would increase the frequency of the LCOs. As illustrated in figure 12(b), some discharges (e.g. \#29310, purple) exhibit larger LCO frequencies with increasing $\nu_{\mathrm{ZF}}$ as expected. All discharges together, however, do not scale with $\nu_{\mathrm{ZF}}$ and especially discharges \#29309 (brown) and \#29315 (yellow) do not follow the trend of the other discharges.

The existence of magnetic precursors as discussed in Section V indicates that the LCOs could be of the same nature as type-III ELMs. As discussed in reference [54], the type-III ELM frequency could be attributed to resistive MHD effects due to its dependence on temperature. Therefore, we compare the LCO frequency with the Spitzer resistivity $\eta_{\text {Spitzer }}$ [46] as shown in figure $12(\mathrm{c})$. The LCO frequency in single discharges predominantly increases with $\eta_{\text {Spitzer }}$ suggesting a positive scaling of the LCO frequency with resistivity. This is in agreement with the property of type-III ELMs which decrease in frequency when the heating power (and therefore temperature) is increased. The frequency scaling of the complete set of discharges, however, is not coherent and, thus, disagrees with a simple dependence of the LCO frequency on resistivity only.

In the so-called M-mode at JET, which shares the higher harmonics in the spectrograms, the $m=1$ magnetic structure and the appearance close to the L-H power threshold with the AUG LCOs, a dependence of the frequency on the poloidal Alfvén speed $v_{A}=$ $B_{\theta} / \sqrt{\mu_{0} m_{i} n}$ was found $[9,10]$. We approximate the poloidal Alfvén speed by the quantity $I_{p} / \sqrt{n}$ and scale it against the measured LCO frequency as shown in figure $12(\mathrm{~d})$. Due to its density dependence, individual discharges clearly scale with $I_{p} / \sqrt{n}$, and at a fixed plasma current of $I_{p}=0.6 \mathrm{MA}$, which was chosen for most of the discharges in the considered data set, the data points lie on top of each other. For discharges with higher plasma current, the frequencies have a clear tendency to be higher. This is in agreement with the proposed M-mode scaling. However, the frequency dependence of individual discharges does not fit to the overall scaling of different discharges with different $I_{p}$ indicating a dependence on further quantities.

The pressure dependence of the LCO frequency as suggested by figure 5(c) could also be related to a perpendicular $E \times B$-flow $v_{E \times B} \sim E_{r} / B \sim \nabla p_{i} /\left(e n_{i} B\right)$, depending on the radial electric field $E_{r}$ which is dominantly determined by the diamagnetic term $\nabla p_{i} /\left(e n_{i} B\right)$ if set by neoclassical processes [18]. A direct comparison of the perpendicular flow velocity with the measured LCO frequency is, however, not possible for the considered data set due to the lack of direct velocity measurements or sufficiently accurate determination of the ion temperatures. Therefore, the density and temperature of the electrons are used instead of $n_{i}$ and $T_{i}$ for the estimation of the pressure gradient terms $\nabla p / n=\nabla T+T \nabla n / n$. While the inverse density gradient length $\nabla n / n$ is evaluated directly from the IDA electron density profiles [45], the temperature gradient is approximated by the finite difference $\left(T_{e}\left(\rho_{\mathrm{pol}}=0.95\right)-T_{e}\left(\rho_{\mathrm{pol}}=1.0\right)\right) / d r$ with constant $T_{e}\left(\rho_{\text {pol }}=1.0\right)=100 \mathrm{eV}$ and $d r=3$ $\mathrm{cm}$. It is conceivable that the LCOs have a constant frequency in the plasma frame, and that they appear at a higher frequency in the lab frame due to the perpendicular motion set by the $E_{r}$ suggesting a scaling $f \sim v_{E \times B} \sim \nabla p_{i} /\left(e n_{i} B\right)$. This scaling, however, cannot be confirmed as shown in figure 12(e) since higher frequencies are found at lower velocities $v_{E \times B}$. In general, the data points from different discharges cluster in a narrow region between $v_{E \times B}=5-10 \mathrm{~km} / \mathrm{s}$, and exhibit no clear trend.

As shown in figure 12(a)-(e), the most of the proposed scalings do not fit to the considered data from different discharges. Therefore, we perform a multivariate linear regression in order to find a best fit of the LCO frequency on its dependent variables. We choose as input $\bar{n}_{\text {e,edge }}$ $\left[10^{19} \mathrm{~m}^{-3}\right], T_{e}[\mathrm{eV}], B_{t}[\mathrm{~T}]$ and $q_{95} . I_{p}$ is not used since it is connected to $B_{t}$ and $q_{95}$ via $q_{95} \sim B_{t} / I_{p}$. The regression yields a frequency dependence

$$
f_{\text {fit }}=976.031 \bar{n}_{\text {e,edge }}^{-1.10 \pm 0.16} T_{e}^{-0.91 \pm 0.07} B_{t}^{1.93 \pm 0.08} q_{95}^{-1.36 \pm 0.08} .
$$

The root mean square error of the fit is 18.99. This scaling can be expressed in terms of $\beta_{\mathrm{t}, \text { ped }}$ and $q_{95}$ with $\beta_{\mathrm{t}, \text { ped }}=2 \mu_{0} e T_{e} \bar{n}_{\mathrm{e}, \text { edge }} / B_{t}^{2}$. It results in an approximate 
scaling

$$
f_{\text {fit }} \sim \frac{1}{\beta_{\mathrm{t}, \mathrm{ped}} q_{95}^{3 / 2}} .
$$

As illustrated in figure $12(\mathrm{f})$, the total data set agrees very well with this approximate scaling and data from very different discharges overlap. Likewise, single discharges mainly follow this scaling and the pressure dependence as discussed in figure $5(\mathrm{c})$ is covered by the proposed scaling. Due to the limitation of measurement accuracy and the small number of data points, the results of the fitting procedure could suggest a misleading trend. Within this limitations, however, our data set exhibits a clear dependence of the LCO frequency on the parameters $\beta_{\mathrm{t} \text {,ped }}$ and $q_{95}$. The fact that these parameters are dimensionless could possibly explain why the LCO frequency is typically found in a narrow range of a few kilohertz in many fusion experiments with very different densities, temperatures and magnetic fields.

\section{DISCUSSION AND SUMMARY}

The following observations during LCOs close to the L$\mathrm{H}$ transition (I-phase) in AUG as presented in this paper have been made:

(1) The LCOs appear (almost) exclusively in favourable configurations with the ion $\mathbf{B} \times \nabla \mathbf{B}$ drift pointing towards the divertor $\mathrm{X}$-point in practically all L- to H-transitions.

(2) In plasmas heated marginally above the L-H power threshold, the LCOs start very regular (early Iphase) and transition into an intermittent or bursty phase (late I-phase). In faster power ramps, only a few of the intermittent type of bursts are present.

(3) At the back transition from H- to L-mode, the same dynamics can be observed, however, in reversed order. LCOs at the back transition from H- to Lmode are observed at higher densities, for which LCOs have not been observed up to now at the L-H transition.

(4) The LCOs are accompanied by an up-down asymmetric magnetic pulsation visible in pick-up coils measuring $\dot{B}_{\theta}$. This pulsation starts to propagate from the divertor regions along the HFS into the ion diamagnetic direction (in lab frame) with a velocity of about $91 \mathrm{~km} / \mathrm{s}$.

(5) The LCO bursts in the late phase are accompanied by precursors clearly visible in the $\dot{B}_{r}$-signal and the reflectometer phase. The precursors fulfill the definition of type-III ELM precursors. Measurements with the reflectometer indicate precursor activity even in the early phase of the LCOs.
(6) During the LCOs, the density profile is modulated starting inside the LCFS and propagating outward with a radial velocity of $100-300 \mathrm{~m} / \mathrm{s}$ into the far scrape-off layer.

(7) The frequency of the LCOs in a limited set of NBIheated AUG discharges scales as

$$
f_{\text {fit }} \sim \frac{1}{\beta_{\mathrm{t}, \text { ped }} q_{95}^{3 / 2}} .
$$

Observation (1) has not been discussed in the context of most of the LCO models although it is a very robust observation. Reference [37] proposes a mechanism which can explain observation (1) based on the assumption that the power threshold from L- to H-mode $P_{\mathrm{LH}}$ differs from the threshold from $\mathrm{H}$ - to L-mode $P_{\mathrm{HL}}$, and that $P_{\mathrm{LH}}$ is higher in unfavourable configuration. Following this idea, the plasma density rises after the $\mathrm{L}-\mathrm{H}$ transition due to better confinement. For a sufficiently high increase of the density, the plasma falls back into L-mode since it reaches $P_{\mathrm{HL}}$ at a certain point. In L-mode the confinement is lower, the density decreases, and the cycle starts from the beginning. If $P_{\mathrm{LH}}$ is too high as it is the case in unfavourable configuration, the cycle cannot be closed since $P_{\mathrm{HL}}$ is never reached and the plasma stays in H-mode. This mechanism only works for a monotonic dependence of $P_{\mathrm{LH}}$ on density and a small difference between $P_{\mathrm{LH}}$ and $P_{\mathrm{HL}}$. For a non-monotonic power threshold as it is found experimentally [47], it is however difficult to understand the appearance of the LCOs in the region of decreasing threshold power $P_{\mathrm{LH}}$ with density in terms of this model. In addition, the model does not explain why $P_{\mathrm{LH}}$ is higher in unfavourable configuration, which is an outstanding question in L-H transition physics. Observation (1) is partially in variance to results from EAST [7] where I-phases were observed in both unfavourable and favourable configuration at the L-H transition. However, on both tokamaks, AUG and EAST, I-phases have been observed at the $\mathrm{H}-\mathrm{L}$ back transition in all configurations. From DIII-D it was reported [6], that the X-point location influences the duration of the I-phase. Symmetry breaking by the poloidal flow direction or influence of local magnetic field quantities $[60,61]$ could generate a configuration dependence inside the LCFS. However, it is not clear whether processes inside the LCFS or the change of SOL conditions are the cause of the configuration dependence of the I-phase.

The intermittency in the late I-phase (observation (2)) was already discussed based on DIII-D data $[5,6]$. While the early I-phase with regular pulsations can be successfully modelled with the majority of the LCO models (especially the model presented in reference [29]), the transition into the intermittent phase is not covered. To our knowledge, the intermittent (or chaotic) state is discussed only in the model derived from MHD equations described in reference [35]. The chaotic solution follows from the structure of the equation system which has similarities 
to the Lorenz model known from chaos theory. Our results imply that the appearance of intermittency in model solutions should be a necessary condition for the correctness of the LCO models. Furthermore, the transition into the intermittent phase could help to shrink the parameter space of the prefactors in the model equations when compared with experimental data.

Observation (3) is in principle covered by all LCO models when the ordering parameter (e.g. heating power) is ramped up and down again. The different role of density and temperature during the forward and back transitions, however, could help to validate the LCO models. The fact, that the I-phase appears always in a similar pressure range, is in line with the observation of a similar $E \times B$ velocity at the onset of the I-phase as suggested by figure $12(\mathrm{e})$. This implies a fixed value of the (shear) flow as a necessary condition for the L-H transition and the onset of the I-phase even for different discharge parameters.

The magnetic data during the I-phase (observation (4)) has the same structure as found in the M-mode at JET $[9,10]$. This suggests that the M-mode from JET is the same as the I-phase at AUG. The results are also similar to the findings during I-phase at EAST [62] and HL-2A [16]. It was proposed that the magnetic $m=1$ structure during the I-phase could simply be a consequence of the reorganisation of Pfirsch-Schlüter flows/currents during modulations of the pressure gradient (cf. observation (6)) according to the MHD equilibrium condition $\mathbf{j} \times \mathbf{B}=\boldsymbol{\nabla} p$ [63]. This interpretation, however, would suggest an inout asymmetry of the magnetic $m=1$-structure. The observation at AUG and EAST [62], however, is an updown asymmetry which cannot be explained by the equilibrium argument. In addition, we found a propagation of the magnetic pulse from the divertor region to the top of the plasma, which points to a non-equilibrium effect. The observation of an up-down asymmetry, however, could be understood in terms of a response of flows and currents on ballooned transport based on time-dependent flux-surface averaged electromagnetic turbulence equations [64].

The magnetic precursors detected in the $\dot{B}_{r}$-signal and in the phase signal of the reflectometer (observation (5)) clearly point to the identity of the late I-phase with typeIII ELMs. In addition, the smooth transition of the regular pulsation state into the intermittent phase, and the indications of weak precursor activity in the early LCO pulses, strongly suggests a common nature of the early and late LCOs. Since it is possible to (qualitatively) reproduce the dynamics of the early and late I-phase with one model [35], the same underlying physical mechanism can -at least in principle- describe the LCO phenomenon and the type-III ELM dynamics. This raises hope to a unified description of the L-H transition, the LCOs and the type-III ELMs with a single reduced model. The magnetic (precursor) activity strongly suggests to extend the established LCO models by electromagnetic or MHD effects since most of them are derived from electrostatic equations. On the experimental side more effort to characterise the structure and role of the precursors in all phases of the LCOs could help to identify the type of instability, which is possibly triggering the LCO burst.

The modulation of the density profile (observation (6)) indicates by causality that the origin of the LCO modulation is located inside the confined region. Strong evidence is found [27] that the modulation of the density gradient impacts the neoclassical radial electric field $E_{\mathrm{r}, \text { neo }}$ during LCOs, which plays an exceptional role in sustaining the $\mathrm{H}$-mode $[7,16,17]$. The radial movement of the pulsation front during LCOs inside the LCFS was also reported from TJ-II [14]. The values of the measured radial velocities agree with our results, however, were estimated from Doppler reflectometry data and can therefore not directly compared with our results obtained from Li-BES data.

The variation of the LCO frequency with different plasma parameters suggests a comparatively simple frequency scaling depending on $\beta_{\mathrm{t}, \text { ped }}$ and $q_{95}$ (observation $(7))$. This is unlike the scaling proposed in reference [34], however, this needs more detailed investigations since we used only rough analytical expressions

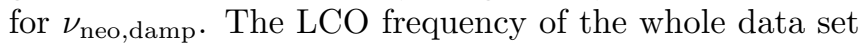
does neither scale with the $E \times B$-flow (figure $12(\mathrm{e})$ ) nor the Spitzer resistivity, although single discharges scale with the resistivity in agreement with the type-III ELM dynamics (figure 12(c)). The observation, that the frequency does not depend on the zonal flow damping rate (figure 12(b)) suggests either that there is no zonal flow activity during the I-phase or that the frequency does not only depend on the damping. The latter is in line with the fact that a classical predator-prey system has a frequency scaling $f \sim \sqrt{\gamma \mu}$ [65] with the growth rate of the prey (turbulence) $\gamma$ and the damping rate of the predator (zonal flow) $\mu \sim \nu_{\mathrm{ZF}}$. From this relation follows a growth rate of the turbulence $\gamma \sim 1 /\left(\beta_{\mathrm{t}, \mathrm{ped}}^{2} q_{95}^{3} \nu_{i i}\right) \sim$ $\left(B_{t} I_{p}^{3}\right) /\left(\bar{n}_{\mathrm{e}, \text { edge }}^{3} T_{e}^{1 / 2}\right)$ if the approximate frequency scaling according to equation 7 and the zonal flow damping $\mu \sim \nu_{\mathrm{ZF}}$ (cf. equation 5) hold. The M-mode frequency scaling $f \sim I_{p} / \sqrt{n}[9,10]$ does likewise not agree well with the considered data set (figure 12(e)). However, the approximate scaling from our fitting suggests $f_{\text {fit }} \sim 1 /\left(\beta_{\mathrm{t}, \text { ped }} q_{95}^{3 / 2}\right) \sim\left(B_{t}^{1 / 2} I_{p}^{3 / 2}\right) /(n T)$ which is quite similar to the M-mode scaling if the magnetic field and temperature dependence is neglected.

In summary, the I-phase in AUG preferentially occurs in favourable magnetic configuration and LCOs (or at least some burst) appear at practically all L-H transitions. We found an up-down asymmetric magnetic activity and indications of precursors of bursts during all phases of the LCOs in AUG plasmas. In particular, the precursor activity points to an electromagnetic nature of the I-phase which has not been taken into account in most of the LCO models. Together with the fact that the LCOs transition smoothly into an intermittent state, the LCO model based on MHD equations [35] qualitatively describes the observations in AUG plasmas best.

Our results indicate that the LCOs during I-phase are 
of the same nature as the type-III ELM phenomenon The LCO frequency for a selected set of AUG discharges scales as $f_{\text {fit }} \sim 1 /\left(\beta_{\text {t,ped }} q_{95}^{3 / 2}\right) \sim\left(B_{t}^{1 / 2} I_{p}^{3 / 2}\right) /(n T)$ which is in variance with existing $\mathrm{LCO}$ frequency scalings $[9,31,34]$.

\section{Acknowledgment}

Many thanks to P. Schneider, R. Dux, C. Angioni, E. Solano and G. Staebler for support and fruitful discus- sions. This work has been carried out within the framework of the EUROfusion Consortium and has received funding from the Euratom research and training programme 2014-2018 under grant agreement No 633053. The views and opinions expressed herein do not necessarily reflect those of the European Commission. F.M. Laggner is a fellow of the Friedrich Schiedel Foundation for Energy Technology.
[1] K. Itoh, S.-I. Itoh, and A. Fujisawa, Plasma and Fusion Research 8, 1102168 (2013).

[2] H. Zohm, Phys. Rev. Lett. 72, 222 (1994).

[3] G. D. Conway, C. Angioni, F. Ryter, P. Sauter, and J. Vicente, Phys. Rev. Lett. 106, 065001 (2011).

[4] S. H. Müller, G. D. Conway, G. Birkenmeier, D. Carralero, T. Happel, A. Herrmann, P. Manz, P. de Marné, A. Mlynek, H. W. Müller, S. Potzel, V. Rohde, U. Stroth, M. Tsalas, G. R. Tynan, E. Wolfrum, and the ASDEX Upgrade Team, Physics of Plasmas 21, (2014).

[5] R. J. Colchin, M. J. Schaffer, B. A. Carreras, G. R. McKee, R. Maingi, T. N. Carlstrom, D. L. Rudakov, C. M. Greenfield, T. L. Rhodes, E. J. Doyle, N. H. Brooks, and M. E. Austin, Phys. Rev. Lett. 88, 255002 (2002).

[6] R. Colchin, B. Carreras, R. Maingi, L. Baylor, T. Jernigan, M. Schaffer, T. Carlstrom, N. Brooks, C. Greenfield, P. Gohil, G. McKee, D. Rudakov, T. Rhodes, E. Doyle, M. Austin, and J. Watkins, Nuclear Fusion 42, 1134 (2002).

[7] G. Xu, H. Wang, M. Xu, B. Wan, H. Guo, P. Diamond, G. Tynan, R. Chen, N. Yan, D. Kong, H. Zhao, A. Liu, T. Lan, V. Naulin, A. Nielsen, J. J. Rasmussen, K. Miki, P. Manz, W. Zhang, L. Wang, L. Shao, S. Liu, L. Chen, S. Ding, N. Zhao, Y. Li, Y. Liu, G. Hu, X. Wu, and X. Gong, Nuclear Fusion 54, 103002 (2014).

[8] Z. Yan, G. R. McKee, R. Fonck, P. Gohil, R. J. Groebner, and T. H. Osborne, Phys. Rev. Lett. 112, 125002 (2014).

[9] E. R. Solano, N. Vianello, P. Buratti, B. Alper, R. Coelho, E. Delabie, S. Devaux, D. Dodt, A. Figueiredo, L. Frassinetti, D. Howell, E. Lerche, C. Maggi, A. Manzanares, A. Martin, J. Morris, S. Marsen, K. McCormick, I. Nunes, D. Refy, F. Rimini, A. Sirinelli, B. Sieglin, S. Zoletnik, and J. E. Contributors, Proceedings of the 40th EPS Conference on Plasma Physics 2013 (EPS, Espoo, Finland, 2013).

[10] N. Vianello and E. R. S. et al., Proceedings of the 42nd EPS Conference on Plasma Physics 2015 (EPS, Lisbon, Portugal, 2015).

[11] Y. Xu, J. Cheng, J. Q. Dong, Y. B. Dong, M. Jiang, W. L. Zhong, L. W. Yan, Z. B. Shi, Z. H. Huang, Y. G. Li, L. Nie, M. Xu, K. J. Zhao, X. Q. Ji, D. L. Yu, Y. Liu, Z. Y. Cui, W. Chen, Q. W. Yang, X. T. Ding, X. R. Duan, and Y. Liu, Plasma Physics and Controlled Fusion 57, 014028 (2015).

[12] T. Kobayashi, K. Itoh, T. Ido, K. Kamiya, S.-I. Itoh, Y. Miura, Y. Nagashima, A. Fujisawa, S. Inagaki, K. Ida, and K. Hoshino, Phys. Rev. Lett. 111, 035002 (2013).

[13] S. J. Zweben, R. J. Maqueda, R. Hager, K. Hallatschek,
S. M. Kaye, T. Munsat, F. M. Poli, A. L. Roquemore, Y. Sechrest, and D. P. Stotler, Physics of Plasmas 17, (2010).

[14] T. Estrada, C. Hidalgo, T. Happel, and P. H. Diamond, Phys. Rev. Lett. 107, 245004 (2011).

[15] M. Hirsch, P. Grigull, H. Wobig, J. Kisslinger, K. McCormick, M. Anton, J. Baldzuhn, S. Fiedler, C. Fuchs, J. Geiger, L. Giannone, H.-J. Hartfuss, E. Holzhauer, M. Hirsch, P. Grigull, H. Wobig, J. Kisslinger, K. McCormick, M. Anton, J. Baldzuhn, S. Fiedler, C. Fuchs, J. Geiger, L. Giannone, H.-J. Hartfuss, R. Jaenicke, M. Kick, H. Maassberg, F. Wagner, A. Weller, and the W7AS Team, Plasma Physics and Controlled Fusion 42, A231 (2000).

[16] J. Cheng, J. Dong, K. Itoh, L. Yan, W. Hong, K. Zhao, Z. Huang, X. Ji, W. Zhong, D. Yu, S.-I. Itoh, L. Nie, X. Song, Q. Yang, X. Ding, X. Zou, X. Duan, Y. Liu, and the HL-2A Team, Nuclear Fusion 54, 114004 (2014).

[17] L. Schmitz, L. Zeng, T. Rhodes, J. Hillesheim, W. Peebles, R. Groebner, K. Burrell, G. McKee, Z. Yan, G. Tynan, P. Diamond, J. Boedo, E. Doyle, B. Grierson, C. Chrystal, M. Austin, W. Solomon, and G. Wang, Nuclear Fusion 54, 073012 (2014).

[18] E. Viezzer, T. Pütterich, C. Angioni, A. Bergmann, R. Dux, E. Fable, R. McDermott, U. Stroth, E. Wolfrum, and the ASDEX Upgrade Team, Nuclear Fusion 54, $012003(2014)$.

[19] Y. H. Xu, C. X. Yu, J. R. Luo, J. S. Mao, B. H. Liu, J. G. Li, B. N. Wan, and Y. X. Wan, Phys. Rev. Lett. 84, 3867 (2000).

[20] P. Manz, G. S. Xu, B. N. Wan, H. Q. Wang, H. Y. Guo, I. Cziegler, N. Fedorczak, C. Holland, S. H. Müller, S. C. Thakur, M. Xu, K. Miki, P. H. Diamond, and G. R. Tynan, Physics of Plasmas 19, (2012).

[21] I. Shesterikov, Y. Xu, G. R. Tynan, P. H. Diamond, S. Jachmich, P. Dumortier, M. Vergote, M. Van Schoor, G. Van Oost, and T. Team, Phys. Rev. Lett. 111, 055006 (2013).

[22] G. Tynan, M. Xu, P. Diamond, J. Boedo, I. Cziegler, N. Fedorczak, P. Manz, K. Miki, S. Thakur, L. Schmitz, L. Zeng, E. Doyle, G. McKee, Z. Yan, G. Xu, B. Wan, H. Wang, H. Guo, J. Dong, K. Zhao, J. Cheng, W. Hong, and L. Yan, Nuclear Fusion 53, 073053 (2013).

[23] I. Cziegler, G. Tynan, P. Diamond, A. Hubbard, J. Hughes, J. Irby, and J. Terry, Nuclear Fusion 55, 083007 (2015).

[24] P. Manz, M. Ramisch, and U. Stroth, Phys. Rev. Lett. 103, 165004 (2009). 
[25] G. Birkenmeier, M. Ramisch, B. Schmid, and U. Stroth, Phys. Rev. Lett. 110, 145004 (2013).

[26] U. Stroth, P. Manz, and M. Ramisch, Plasma Physics and Controlled Fusion 53, 024006 (2011).

[27] M. Cavedon et al., to be published.

[28] P. H. Diamond and Y. B. Kim, Phys. Fluids, B 3, 1626 (1991).

[29] E.-J. Kim and P. H. Diamond, Phys. Rev. Lett. 90, 185006 (2003).

[30] M. Sasaki, K. Itoh, S.-I. Itoh, and N. Kasuya, Nuclear Fusion 52, 023009 (2012).

[31] K. Miki, P. H. Diamond, Ö. D. Gürcan, G. R. Tynan, T. Estrada, L. Schmitz, and G. S. Xu, Physics of Plasmas 19, 092306 (2012).

[32] M. A. Malkov, P. H. Diamond, K. Miki, J. E. Rice, and G. R. Tynan, Physics of Plasmas 22, (2015).

[33] S.-I. Itoh, K. Itoh, A. Fukuyama, and Y. Miura, Phys. Rev. Lett. 67, 2485 (1991).

[34] G. M. Staebler and R. J. Groebner, Plasma Physics and Controlled Fusion 57, 014025 (2015).

[35] D. Constantinescu, O. Dumbrajs, V. Igochine, K. Lackner, R. Meyer-Spasche, H. Zohm, and A. U. Team, Physics of Plasmas 18, (2011).

[36] J. W. Connor, Plasma Phys. Controll. Fusion 40, 191 (1998).

[37] H. Zohm, W. Suttrop, K. Buchl, H. J. de Blank, O. Gruber, A. Kallenbach, V. Mertens, F. Ryter, and M. Schittenhelm, Plasma Physics and Controlled Fusion 37, 437 (1995).

[38] S. K. Rathgeber, L. Barrera, T. Eich, R. Fischer, B. Nold, W. Suttrop, M. Willensdorfer, E. Wolfrum, and the ASDEX Upgrade Team, Plasma Physics and Controlled Fusion 55, 025004 (2013).

[39] G. D. Conway, B. Scott, J. Schirmer, M. Reich, A. Kendl, and the ASDEX Upgrade Team, Plasma Phys. Controll. Fusion 47, 1165 (2005).

[40] G. D. Conway, E. Poli, T. Happel, and the ASDEX Upgrade Team, Plasma and Fusion Research 5, S2005 (2010).

[41] R. Fischer, E. Wolfrum, J. Schweinzer, and the ASDEX Upgrade Team, Plasma Physics and Controlled Fusion 50, 085009 (2008).

[42] M. Willensdorfer, G. Birkenmeier, R. Fischer, F. M. Laggner, E. Wolfrum, G. Veres, F. Aumayr, D. Carralero, L. Guimarais, B. Kurzan, and ASDEX Upgrade Team, Plasma Physics and Controlled Fusion 56, 025008 (10pp) (2014).

[43] M. Willensdorfer, E. Wolfrum, A. Scarabosio, F. Aumayr, R. Fischer, B. Kurzan, R. McDermott, A. Mlynek, B. Nold, S. Rathgeber, V. Rohde, F. Ryter, P. Sauter, E. Viezzer, and the ASDEX Upgrade Team, Nuclear Fusion 52, 114026 (2012).

[44] T. Happel, P. Manz, F. Ryter, P. Hennequin, A. Hetzenecker, G. Conway, C. Honoré, U. Stroth, E. Viezzer, and the ASDEX Upgrade Team, Nuclear Fusion 56, 064004 (2016)

[45] R. Fischer, C. J. Fuchs, B. Kurzan, W. Suttrop, E. Wolfrum, and A. U. Team, Fusion Science and Technology
58, $675(2010)$

[46] J. Wesson, Tokamaks (Clarendon Press, Oxford, 1987).

[47] F. Ryter, S. Rathgeber, L. B. Orte, M. Bernert, G. Conway, R. Fischer, T. Happel, B. Kurzan, R. McDermott, A. Scarabosio, W. Suttrop, E. Viezzer, M. Willensdorfer, E. Wolfrum, and the ASDEX Upgrade Team, Nuclear Fusion 53, 113003 (2013).

[48] J. C. van den Berg, Wavelets in Physics (Cambridge University Press, Cambridge, 1999).

[49] L. Cupido, S. Graca, G. D. Conway, M. Manso, F. Serra, and A. U. Team, Review of Scientific Instruments 77, (2006).

[50] G. D. Conway, Plasma Physics and Controlled Fusion 41, 65 (1999).

[51] J. Vicente, G. D. Conway, M. E. Manso, H. W. Müller, C. Silva, F. da Silva, L. Guimarais, and A. Silva, Plasma Physics and Controlled Fusion 56, 125019 (2014).

[52] D. Prisiazhniuk, A. Kraemer-Flecken, G. Conway, P. Manz, T. Happel, P. Simon, U. Stroth, and the ASDEXUpgrade Team, 12th International Reflectometry Workshop (IRW12), FZ-Jülich, Germany, 2015 (FZJ, FZJülich, 2015).

[53] H. Zohm, F. Wagner, M. Endler, J. Gernhardt, E. Holzhauer, W. Kerner, and V. Mertens, Nuclear Fusion 32, 489 (1992).

[54] H. Zohm, Plasma Physics and Controlled Fusion 38, 105 (1996).

[55] B. Kurzan and H. D. Murmann, Review of Scientific Instruments 82, (2011).

[56] Y. B. Kim, P. H. Diamond, and R. J. Groebner, Physics of Fluids B 3, (1991).

[57] O. Sauter, C. Angioni, and Y. R. Lin-Liu, Physics of Plasmas 6, (1999).

[58] Z. Lin, T. S. Hahm, W. W. Lee, W. M. Tang, P. H. Diamond, Phys. Rev. Lett. 83, 3645 (1999)

[59] P. H. Diamond, S.-I. Itoh, K. Itoh, and T. S. Hahm, Plasma Phys. Controll. Fusion 47, R35 (2005).

[60] F. Alladio, F. Crisanti, A. Mancuso, P. Micozzi, O. Tudisco, K. H. Burrell, M. S. Chu, L. L. Lao, T. H. Osborne, R. Giannella, P. Lomas, D. O'Brien, V. Riccardo, and B. Schunke, 6, 2472 (1999).

[61] G. Birkenmeier, M. Ramisch, P. Manz, B. Nold, and U. Stroth, Phys. Rev. Lett. 107, 025001 (2011).

[62] G. Xu, L. Shao, S. Liu, H. Wang, B. Wan, H. Guo, P. Diamond, G. Tynan, M. Xu, S. Zweben, V. Naulin, A. Nielsen, J. J. Rasmussen, N. Fedorczak, P. Manz, K. Miki, N. Yan, R. Chen, B. Cao, L. Chen, L. Wang, W. Zhang, and X. Gong, Nuclear Fusion 54, 013007 (2014).

[63] J. Cheng, J. Q. Dong, K. Itoh, L. W. Yan, M. Xu, K. J. Zhao, W. Y. Hong, Z. H. Huang, X. Q. Ji, W. L. Zhong, D. L. Yu, S.-I. Itoh, L. Nie, D. F. Kong, T. Lan, A. D. Liu, X. L. Zou, Q. W. Yang, X. T. Ding, X. R. Duan, and Y. Liu, Phys. Rev. Lett. 110, 265002 (2013).

[64] P. Manz, submitted to Physics of Plasmas (2015).

[65] M. Arrigoni and A. Steiner, Acta Biotheoretica 32, 123 (1983). 